\title{
Saturnin: a suite of lightweight symmetric algorithms for post-quantum security*
}

\author{
Anne Canteaut ${ }^{1}$, Sébastien Duval ${ }^{2}$, Gaëtan Leurent ${ }^{1}$, María Naya-Plasencia ${ }^{1}$, \\ Léo Perrin ${ }^{1}$, Thomas Pornin ${ }^{3}$ and André Schrottenloher ${ }^{1}$ \\ ${ }^{1}$ Inria, Paris, France, \{anne.canteaut, gaetan.leurent, maria.naya_plasencia, leo.perrin, \\ andre.schrottenloher\}@inria.fr \\ ${ }^{2}$ UCL Crypto Group, Louvain-la-Neuve, Belgium, sebastien.pf .duval@gmail.com \\ ${ }^{3}$ NCC Group, Quebec, Canada, Thomas.pornin@nccgroup.com
}

\begin{abstract}
The cryptographic algorithms needed to ensure the security of our communications have a cost. For devices with little computing power, whose number is expected to grow significantly with the spread of the Internet of Things (IoT), this cost can be a problem. A simple answer to this problem is a compromise on the security level: through a weaker round function or a smaller number of rounds, the security level can be decreased in order to cheapen the implementation of the cipher. At the same time, quantum computers are expected to disrupt the state of the art in cryptography in the near future. For public-key cryptography, the NIST has organized a dedicated process to standardize new algorithms. The impact of quantum computing is harder to assess in the symmetric case but its study is an active research area.

In this paper, we specify a new block cipher, SATURNIN, and its usage in different modes to provide hashing and authenticated encryption in such a way that we can rigorously argue its security in the post-quantum setting. Its security analysis follows naturally from that of the AES, while our use of components that are easily implemented in a bitsliced fashion ensures a low cost for our primitives. Our aim is to provide a new lightweight suite of algorithms that performs well on small devices, in particular micro-controllers, while providing a high security level even in the presence of quantum computers.

SATURNIN is a 256-bit block cipher with a 256-bit key and an additional 9-bit parameter for domain separation. Using it, we built two authenticated ciphers and a hash function.
\end{abstract}

- SAturnin-CTR-Cascade is an authenticated cipher using the counter mode and a separate MAC. It requires two passes over the data but its implementation does not require the inverse block cipher.

- SATURnin-Short is an authenticated cipher intended for messages with a length strictly smaller than 128 bits which uses only one call to SATURNIN to provide confidentiality and integrity.

- SAturnin-Hash is a 256-bit hash function.

In this paper, we specify this suite of algorithms and argue about their security in both the classical and the post-quantum setting.

Keywords: lightweight cryptography ' post-quantum security · block cipher · authenticated encryption · hash function · AES · duck

*https://project.inria.fr/saturnin/ 


\section{Introduction}

The aim of SATURNIN is to provide a lightweight suite of algorithms that performs well on small devices and that provides a high security against quantum adversaries.

\subsection{Post-quantum Symmetric Cryptography}

Quantum computation was first introduced in the late $80 \mathrm{~s}$ as a general framework and potential tool for simulating quantum systems. Since then, it has been the subject of much more attention in computer science since the introduction by Shor [Sho94] of a quantum algorithm for solving factorization and discrete logarithms in polynomial time. Since then, the cryptographic community has been concerned with the impact of large or intermediate-scale quantum computers which, although they are yet to be built, would have massive consequences on the currently deployed public-key cryptosystems, breaking most of those that are in use today.

It is widely acknowledged that new cryptographic designs should take into account the quantum threat. As examples of this new direction, one may cite the NIST post-quantum standardization project [Nat16], which structures most of the efforts of the asymmetric cryptographic community, or the report of the National Academies of Sciences [Nat18], which gives a precise evaluation of the quantum threat, up to the uncertainties inherent to the evolution of cutting-edge technologies.

Until recently, such concerns did not seem to apply to symmetric cryptography, which does not rely on structured mathematical problems such as factorization. In contrast, Grover's algorithm [Gro96] provides a quadratic speedup for a wide range of exhaustive search problems, which are relevant to symmetric cryptography. In particular, such a speedup occurs when performing an exhaustive search for secret keys, which brings the cost of this search from $2^{128}$ encryptions to its square root, $2^{64}$ operations, in the case of a 128-bit keyed block cipher such as AES-128. This leads to the natural countermeasure of increasing key sizes, as the report [Nat18] indicates:

"Even if a computer existed that could run Grover's algorithm to attack AES-GCM, the solution is quite simple: increase the key size of AES-GCM from 128-bit to 256-bit keys. "

This indeed brings the cost back to $2^{128}$.

Many works have dealt with the precise complexity of Grover's search for practical attacks, which may be more difficult to run in practice than the first estimate of $2^{64}$ time. For example, one needs to implement symmetric cryptographic operations on a quantum computer, and this is not trivial (see for instance the careful evaluation of applying Grover on AES from [GLRS16]). But, as the report [Nat18] also mentions, this exhaustive search for the key is only an upper bound on the security, not a lower bound; more analysis is needed to assess the security of AES (or other ciphers) against quantum computers.

"More precisely, it is possible that there is some currently unknown clever quantum attack on AES-GCM that is far more efficient than Grover's algorithm."

Recent works have shed a new light on the quantum security (or insecurity) of some symmetric cryptographic constructions [KLLN16a, CNS17, BNPS19]. In general, it can be studied in the following two models defined e.g. in [Gag17, GHS16, KLLN16b].

Q1 Model. A first natural question is the security with an offline quantum computer. While the adversary can only recover classical data, for instance by making queries to a secret-key oracle, she can run quantum computations. Secret-key exhaustive search 
using Grover's algorithm runs in this model, as the adversary only needs a few classical plaintext-ciphertext queries to check her key guesses. Quantum collision search for hash functions runs in this model, as the specification of a hash function is public, and so a quantum adversary is perfectly capable of implementing this function as a quantum circuit.

Q2 Model. The Q2 model is a strictly more powerful setting for the adversary, since the adversary is allowed to perform quantum superposition access to secret-key oracles. There exist classical primitives enjoying a classical security proof, for example the Even-Mansour cipher with a random permutation, which are broken in the Q2 model in polynomial time [KM10, KM12]. In [KLLN16a], it was shown that such attacks could actually target many modes of operation, and accelerate exponentially some classical slide attacks, which concern ciphers with a repetitive structure.

This is a powerful model, but there are many good reasons to consider it, like for instance that it is simple and easy to define; that it is non-trivial, as many primitives and constructions remain resistant in this setting; and also the fact that it includes all possible intermediate scenarios, like the ones were the primitives could be implemented in no-safe manners as components of more complex protocols that might include obfuscation, or implementations on hybrid systems. Countering these attacks using ad hoc methods, for instance by enforcing an input measure that would make the superposition collide, does not seem easy to guarantee nor simple to implement for now. Due to all these reasons, Q2 is the most-widely used model in quantum security proofs, e.g., for quantum proofs of MAC constructions [BZ13b, AMRS], and we have chosen to build primitives offering security in this model.

Let us point out that, though the direct applicability of attacks in this model might be controversial, a substantial part of the community agrees that considering it for defining the security of a system is interesting as it covers security in all the possible intermediate scenarios.

Throughout this paper, whenever we consider an oracle such as a secret-key encryption or decryption oracle, or a tag verification oracle, we consider a quantum adversary to have access to the corresponding Q2 oracle, and our quantum security claims are all in this model.

Other Limitations. Some classically efficient algorithms do not enjoy a clear quantum speedup and, in particular, their quantum versions may encounter new hardware limitations. Such an example is quantum collision search. For an $n$-bit random function, classical collision search runs in time $O\left(2^{n / 2}\right)$, corresponding to the birthday bound, and in $O(n)$ memory thanks to Pollard's rho method. A quantum collision search algorithm found in 1998 [BHT98] reached a complexity of $O\left(2^{n / 3}\right)$ time and queries for the same problem, later proven to be optimal. However, it requires $2^{n / 3}$ quantum memory, so a more than significant amount of hardware. To date, no quantum algorithm optimal in time and as efficient in hardware as Pollard's rho exists for this problem. A tradeoff was given in [CNS17] with a suboptimal time complexity of $2^{2 n / 5}$, but only $O(n)$ quantum memory, showing that quantum collision search should not be immediately ruled out because of its apparent impracticality. The most conservative approach should take $2^{n / 3}$ as a quantum security level, but we choose later to give a bound depending on the amount of quantum memory available to our offline adversary. Additionally, some quantum algorithms such as Grover's encounter inherent difficulties at parallelization. We choose to remain conservative in this setting and consider only a quantum time complexity for a single processor. We aim at a resistance against quantum adversaries even allowing them to use big quantities of quantum memory. 


\subsection{Lightweight and Post-quantum: towards Saturnin}

In the report on lightweight cryptography NISTIR $8114^{1}$, it was explicitly asked that the algorithms submitted to the project should be quantum-safe when long-term security is needed:

"When long-term security is needed, these algorithms should either
aim for post-quantum security, or the application should allow them
to be easily replaceable by algorithms with post-quantum security."

This completely makes sense, as the effort for recommendations for post-quantum asymmetric cryptography is being made, and this will only be effective if the symmetric cryptography used with it is also quantum-resistant.

However, to achieve an effective quantum security of 128 bits with a block cipher, it does not seem enough to use a 256-bit key-size. Indeed, the security of most modes of operation is limited by the complexity of finding collisions, which may benefit from quantum acceleration, depending only on the block size. This yields a first challenge: the design of a block cipher with a bigger block size (we will take 256 bits).

A second challenge is motivated by the results from [KLLN16a] which show that most authentication modes suffer from polynomial-time attacks in the Q2 model. Whether these attacks could be avoided was an open question related to the influence of the nonce on the different calls to the block cipher.

A third challenge is that of lightness: it is not sufficient that our algorithm be postquantum secure, it should also be suitable for efficient implementation on devices with little computing power.

In this proposal we overcome all those issues first by designing a block cipher with a 256bit block size and 256-bit key that inherits the AES security properties while allowing an efficient bitsliced implementation, and secondly by proposing modes of operation resisting the aforementioned attacks.

How to efficiently build an AES with a 256-bit state? Our proposal, SATURNIN is a suite of lightweight symmetric algorithms for post-quantum security. As previously explained, our first aim was to design a block cipher, not only with a key-size equal to twice the desired security level, but also a double state-size. SATURNIN therefore works on blocks of 256 bits, yet also aims at being particularly suitable for high-security microcontroller applications, thanks to an efficient bitslice implementation. Any circuit can be implemented in the bitslice format, as shown by Biham in the case of DES[Bih97], and (much later on) by Matsui and Nakajima for AES[MN07]. Some cryptographic algorithms have been specially designed to better fit such implementation strategies, notably in an internal way in which parallelism within a single instance is exploited; some examples of such algorithms are 3-Way[DGV94] and Serpent[BAK98]. Intuitively, algorithms amenable to efficient bitslice implementations will be adequate for optimized hardware (ASIC, FPGA). We therefore wanted to design SATURNIN along similar lines.

We also wanted to take advantage of the knowledge obtained from the AES analysis and the wide-trail strategy [Dae95], since the AES can be safely considered as the most analyzed block cipher. Indeed, in order to benefit from this 20 year-long cryptanalysis effort, we have built a 3-dimensional AES, on which the strongly-aligned version of the wide-trail strategy [DR02a] can still be applied.

On larger versions of Rijndael. The Rijndael family of block ciphers [DR99] includes larger-state versions, that were not kept in the AES standard. But for larger block-sizes, reaching full diffusion within the internal state is slower since the state is represented, in almost all versions, by a rectangle and not a square. This slower diffusion was exploited

\footnotetext{
${ }^{1}$ https://nvlpubs.nist.gov/nistpubs/ir/2017/NIST.IR.8114.pdf
} 
in several attacks, taking advantage of the larger internal state [GM08, MPP09, NP07, $\mathrm{ZWP}^{+} 08, \mathrm{WGR}^{+} 13$, Sas10]. The long-key versions also seem relatively less secure than the versions using shorter keys, especially regarding related-key attacks [BKN09, BK09]. Moreover, from an implementation point of view, their performances make them less competitive than the version with a 128-bit block size defined in the AES standard.

Previous attempts. The idea of an AES version with three dimensions was mentioned in [DR02b], without further development. In 2008 the block cipher 3D was proposed by Nakahara Jr. in [Nak08]. SATURNin follows an overall approach reminiscent of 3D in that it defines a 3D AES-like block cipher which uses similar subroutines. However, SATURNIN differs from $3 \mathrm{D}$ in at least two key ways. First, the order in which we apply these operations differs. This allows us to claim 125 active S-boxes for 8 rounds, unlike in 3D. Furthermore, all of our operations are intended for an efficient bitsliced implementation, meaning that our cipher is much more suited for the lightweight context.

Some hash function proposals based on the AES transformations with a large internal state were also submitted to the SHA-3 competition, like LANe [IAC $\left.{ }^{+} 08\right]$, Grøstl [GKM ${ }^{+} 08$ ], and $\mathrm{ECHO}\left[\mathrm{GBB}^{+} 08\right]$. However, in each case, the approach used to handle a larger state is different: LANE uses several independent AES states, Grøstl uses a large MDS matrix, and ECHO resembles a four-dimensional AES, but the wide-trail arguments only go through three dimensions. ${ }^{2}$

\subsection{Security claims}

We claim that, for each element in the SATURNIN suite, there is no attack significantly better than the generic attacks against the corresponding construction. In Section 3 we formulate specific security claims taking into account the requirements mentioned in the NIST call for submissions, and we also formulate security claims regarding quantum adversaries in the different models we just introduced.

\section{Specification}

\subsection{The Block Cipher Saturnin}

SATURNin operates on 256-bit blocks, using a 256-bit key. It uses a 256-bit internal state. Blocks, keys and state values can be viewed as several equivalent representations:

- as a $4 \times 4 \times 4$ cube of 4 -bit nibbles (Figure $1 \mathrm{a}$ );

- as sixteen 16-bit registers, indexed from 0 to 15, known as the "bitsliced representation" (Figure 1b);

- as 256 bits.

In this section, we use the first (i.e. 3-dimensional) representation as a cube of nibbles. Section 4 will describe the (2-dimensional) bitsliced representation, and how conversions are performed between all three representations. Practical implementations are expected to use the bitsliced representation; hence, the description in this section is only for formal presentation.

The nibbles in the cube are numbered from 0 to 63, as on Figure 1a. Each nibble can also be defined via coordinates $(x, y, z)$ such that the coordinates $(x, y, z)$ correspond to the nibble with index $(y+4 x+16 z)$. We number the bits within each nibble from 0 to 3 , where 0 is the least significant bit.

\footnotetext{
${ }^{2}$ The bounds given in $\left[\mathrm{GBB}^{+} 08\right]$ show at least 200 active S-Boxes for 8 S-box layers, but a 4D AES would have 625 active S-Boxes for 16 S-box layers.
} 


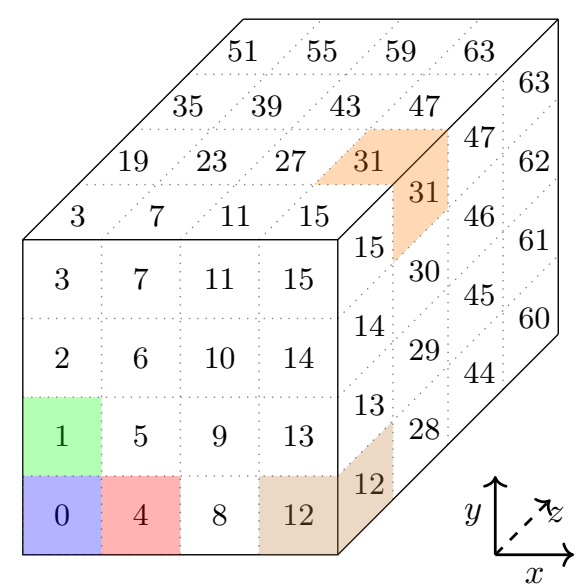

(a) As a $4 \times 4 \times 4$ cube of 4 -bit nibbles. The boundaries between the nibbles are in gray.

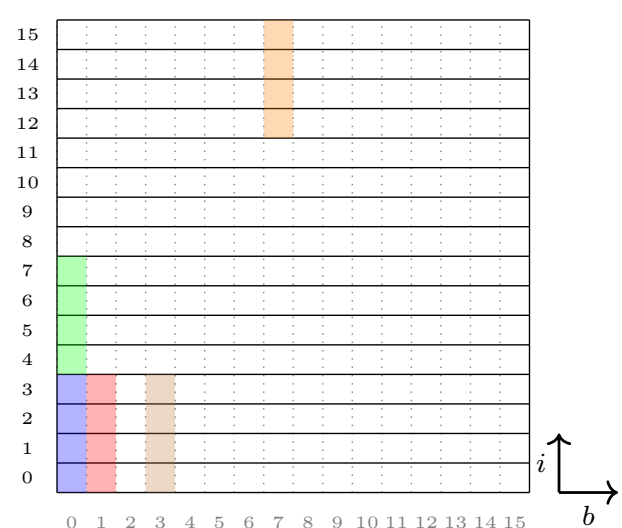

(b) As sixteen 16-bit registers. The indices and boundaries of the registers are in black, those of the bits are in gray.

Figure 1: The 3-dimensional and 2-dimensional representations of the 256-bit state of SATURnin. Nibbles and their corresponding bits are represented with the same color in each representation.

\subsubsection{Internal State}

The various transformations in SATURNIN are defined over different subsets of its state. Below, we define three such subsets of the cube, based on the same terminology as for parts of the Keccak-f state in SHA-3 (see Figure 1.1 in [BDPA11]).

Slice. In the cube, a slice is a subset of the nibbles such that $z$ is constant.

Sheet. In the cube, a sheet is a subset of the nibbles such that $x$ is constant.

Columns. Columns are the intersection of a sheet and a slice. They correspond to the sets of nibbles with $x$ and $z$ constant.

\subsubsection{The Specification of the Block Cipher}

The SATURNIN block cipher is an SPN (in the broadest sense) with an even number of rounds, numbered from 0 . In the following, we call a super-round the composition of two consecutive rounds with indices $2 r$ and $(2 r+1)$.

The SATURNIN block cipher uses a 256-bit internal state $\mathrm{X}$ and a 256-bit key state K, both represented as a $(4 \times 4 \times 4)$-cube of nibbles. Two additional 16 -bit words $\mathrm{RC}_{0}$ and $\mathrm{RC}_{1}$ are used for generating the successive round constants. Pseudo-code describing a full block encryption is given in Algorithm 1.

Parameters. The block cipher has two input parameters:

- $R$ : the number of super-rounds, i.e. the total number of rounds divided by 2 . $R$ belongs to $\{10, \ldots, 31\}$ and is equal to 10 by default.

- D: a 4-bit value, named the domain separator, depending on the operating mode as specified in Section 2.5. The block cipher with domain separator $D$ will be denoted by SATURNiN $^{D}$.

The block cipher with $R$ super-rounds and Domain separator $D$ is denoted by $\operatorname{SATURNIN}_{R}^{D}$. 
Initialization. $\mathrm{X}$ and $\mathrm{K}$ are respectively initialized with the input and with the master key. Both 16-bit registers $\mathrm{RC}_{0}$ and $\mathrm{RC}_{1}$ are initialized as the bit-string

$$
\underbrace{1 \ldots 1}_{7 \text { ones }} \underbrace{R_{4} \ldots R_{0}}_{R} \underbrace{D_{3} \ldots D_{0}}_{D}
$$

where the rightmost bit of the register is the least significant bit. The first four bits are given by the domain separator $\sum_{i=0}^{3} D_{i} 2^{i}=D$, while the 5 -bit integer $\sum_{i=0}^{4} R_{i} 2^{i}$ is equal to $R$, i.e. to the number of super-rounds.

Round 0 starts by xoring $\mathrm{K}$ to the internal state.

Round function. Each round, starting from Round 0, then successively applies the following transformations to the internal state:

- An S-box layer S, which applies the same 4-bit S-box $\sigma_{0}$ to all nibbles with an even index, and the same 4-bit S-box $\sigma_{1}$ to all nibbles with an odd index. These two S-boxes are defined by their lookup tables which are given in Table 1, where $x$ such that $\sum_{i=0}^{3} x_{i} 2^{i}=x$ corresponds to a nibble containing $\left(x_{3}, x_{2}, x_{1}, x_{0}\right)$. An efficient implementation of the S-boxes is shown in Figure 2.

- A nibble permutation $\mathrm{SR}_{r}$ which depends on the round number $r$. For all even rounds, $\mathrm{SR}_{r}$ is the identity function. For odd rounds of index $r$ with $r \bmod 4=1$, $\mathrm{SR}_{r}=\mathrm{SR}_{\text {slice }}$ consists of the parallel application of $R_{\text {slice }}$ on each slice independently. This operation maps the nibble with coordinates $(x, y, z)$ to $(x+y \bmod 4, y, z)$. For odd rounds of index $r$ with $r \bmod 4=3, \mathrm{SR}_{r}=\mathrm{SR}_{\text {sheet }}$ consists of the parallel application of $R_{\text {sheet }}$ on each sheet independently. This operation maps the nibble with coordinates $(x, y, z)$ to $(x, y, z+y \bmod 4)$. The $\mathrm{SR}_{r}$ transformation is depicted on Figure 4.

- A linear layer MC composed of 16 copies of a linear operation $M$ over $\left(\mathbb{F}_{2}^{4}\right)^{4}$ which is applied in parallel to each column of the internal state. The transformation $M$ is defined as:

$$
M:\left(\begin{array}{l}
a \\
b \\
c \\
d
\end{array}\right) \mapsto\left(\begin{array}{c}
\alpha^{2}(a) \oplus \alpha^{2}(b) \oplus \alpha(b) \oplus c \oplus d \\
a \oplus \alpha(b) \oplus b \oplus \alpha^{2}(c) \oplus c \oplus \alpha^{2}(d) \oplus \alpha(d) \oplus d \\
a \oplus b \oplus \alpha^{2}(c) \oplus \alpha^{2}(d) \oplus \alpha(d) \\
\alpha^{2}(a) \oplus a \oplus \alpha^{2}(b) \oplus \alpha(b) \oplus b \oplus c \oplus \alpha(d) \oplus d
\end{array}\right)
$$

where $a$ is the nibble with the lowest index, and $\alpha$ transforms the four bits $x_{0}, \ldots, x_{3}$ of each nibble by the following multiplication

$$
\left(\begin{array}{llll}
0 & 1 & 0 & 0 \\
0 & 0 & 1 & 0 \\
0 & 0 & 0 & 1 \\
1 & 1 & 0 & 0
\end{array}\right)\left(\begin{array}{l}
x_{0} \\
x_{1} \\
x_{2} \\
x_{3}
\end{array}\right)
$$

This transformation corresponds to the next-state function of an LFSR of length 4, in Fibonacci mode, with feedback polynomial $X^{4}+X^{3}+1$.

The transformation $M$ can be implemented efficiently as depicted on Figure 3 (which corresponds to Figure 13 in [DL18] up to a rotation of the nibbles).

- The inverse of the previous nibble permutation, namely $\mathrm{SR}_{r}^{-1}$.

- A sub-key addition at odd rounds only (i.e. at the end of each super-round). The sub-key is composed of the XOR of a round constant and either the master key or a rotated version of the master key: 
Round constant. The round constants $\mathrm{RC}_{0}$ and $\mathrm{RC}$ are updated by clocking 16 times two independent LFSR of length 16 in Galois mode with respective feedback polynomial $X^{16}+X^{5}+X^{3}+X^{2}+1$ and $X^{16}+X^{6}+X^{4}+X+1$. In other words, we repeat 16 times the following operation: if the most significant bit of $\mathrm{RC}_{i}$ is $0, \mathrm{RC}_{i}$ is replaced by $\mathrm{RC}_{i} \ll 1$, otherwise, it is replaced by

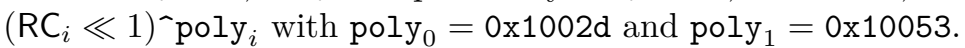

The two 16-bit words $\mathrm{RC}_{0}, \mathrm{RC}_{1}$ are then xored to the internal state. Bit number $i$ in $\mathrm{RC}_{0}$ is added to Bit 0 of the nibble with index $4 i$, for $0 \leq i \leq 15$. Similarly, Bit number $i$ in $\mathrm{RC}_{1}$ is added to Bit 0 of the nibble with index $(4 i+2)$, for $0 \leq i \leq 15$.

Round key. If the round index $r$ is such that $r \bmod 4=3$, the master key $\mathrm{K}$ is xored to the internal state; otherwise (i.e. when $r \bmod 4=1$ ), a rotated version of the key is added instead: the nibble with index $i$ receives the key nibble with index $(i+20) \bmod 64$, for $0 \leq i \leq 63$.

Table 1: The lookup tables of the S-boxes we use.

\begin{tabular}{ccccccccccccccccc}
\hline$x$ & 0 & 1 & 2 & 3 & 4 & 5 & 6 & 7 & 8 & 9 & 10 & 11 & 12 & 13 & 14 & 15 \\
\hline$\sigma_{0}(x)$ & 0 & 6 & 14 & 1 & 15 & 4 & 7 & 13 & 9 & 8 & 12 & 5 & 2 & 10 & 3 & 11 \\
$\sigma_{1}(x)$ & 0 & 9 & 13 & 2 & 15 & 1 & 11 & 7 & 6 & 4 & 5 & 3 & 8 & 12 & 10 & 14 \\
\hline
\end{tabular}

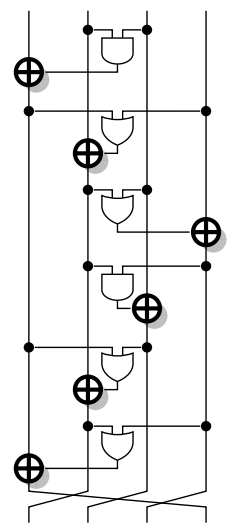

(a) $\sigma_{0}$

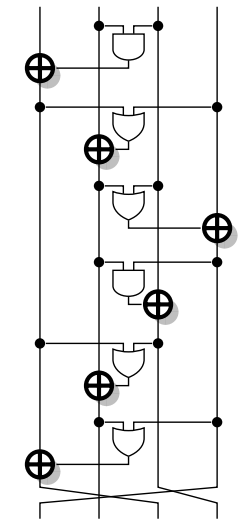

(b) $\sigma_{1}$

Figure 2: Bitslice implementation of the S-box layer. 


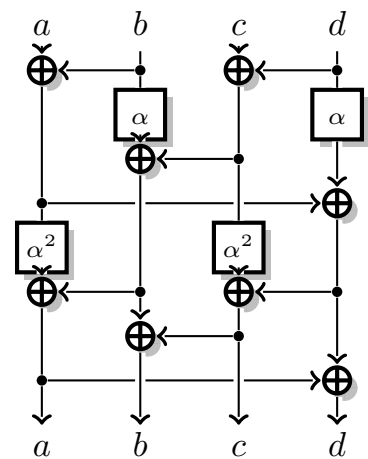

Figure 3: A 4-bit MDS mapping from [DL18]. The input/output $(a, b, c, d)$ corresponds to nibbles with index $(4 i, 4 i+1,4 i+2,4 i+3)$, for $0 \leq i \leq 15$.

Initial state:

\begin{tabular}{|c|c|c|c|}
\hline 3 & 7 & 11 & 15 \\
\hline 2 & 6 & 10 & 14 \\
\hline 1 & 5 & 9 & 13 \\
\hline 0 & 4 & 8 & 12 \\
\hline
\end{tabular}

\begin{tabular}{|l|l|l|l|}
\hline 19 & 23 & 27 & 31 \\
\hline 18 & 22 & 26 & 30 \\
\hline 17 & 21 & 25 & 29 \\
\hline 16 & 20 & 24 & 28 \\
\hline
\end{tabular}

\begin{tabular}{|l|l|l|l|}
\hline 35 & 39 & 43 & 47 \\
\hline 34 & 38 & 42 & 46 \\
\hline 33 & 37 & 41 & 45 \\
\hline 32 & 36 & 40 & 44 \\
\hline
\end{tabular}

\begin{tabular}{|l|l|l|l|}
\hline 51 & 55 & 59 & 63 \\
\hline 50 & 54 & 58 & 62 \\
\hline 49 & 53 & 57 & 61 \\
\hline 48 & 52 & 56 & 60 \\
\hline
\end{tabular}

Internal state after $\mathrm{SR}_{r}$ at Rounds $r$ with $r \equiv 1 \bmod 4$, i.e. after $\mathrm{SR}_{\text {slice }}$ :

\begin{tabular}{|c|c|c|c|}
\hline 7 & 11 & 15 & 3 \\
\hline 10 & 14 & 2 & 6 \\
\hline 13 & 1 & 5 & 9 \\
\hline 0 & 4 & 8 & 12 \\
\hline
\end{tabular}

\begin{tabular}{|l|l|l|l|}
\hline 23 & 27 & 31 & 19 \\
\hline 26 & 30 & 18 & 22 \\
\hline 29 & 17 & 21 & 25 \\
\hline 16 & 20 & 24 & 28 \\
\hline
\end{tabular}

\begin{tabular}{|l|l|l|l|}
\hline 39 & 43 & 47 & 35 \\
\hline 42 & 46 & 34 & 38 \\
\hline 45 & 33 & 37 & 41 \\
\hline 32 & 36 & 40 & 44 \\
\hline
\end{tabular}

\begin{tabular}{|l|l|l|l|}
\hline 39 & 43 & 47 & 35 \\
\hline 42 & 46 & 34 & 38 \\
\hline 45 & 33 & 37 & 41 \\
\hline 32 & 36 & 40 & 44 \\
\hline
\end{tabular}

Internal state after $\mathrm{SR}_{r}$ at Rounds $r$ with $r \equiv 3 \bmod 4$, i.e. after $\mathrm{SR}_{\text {sheet }}$ :

\begin{tabular}{|c|c|c|c|}
\hline 19 & 23 & 27 & 31 \\
\hline 34 & 38 & 42 & 46 \\
\hline 49 & 53 & 57 & 61 \\
\hline 0 & 4 & 8 & 12 \\
\hline
\end{tabular}

\begin{tabular}{|c|c|c|c|}
\hline 35 & 39 & 43 & 47 \\
\hline 50 & 54 & 58 & 62 \\
\hline 1 & 5 & 9 & 13 \\
\hline 16 & 20 & 24 & 28 \\
\hline
\end{tabular}

\begin{tabular}{|c|c|c|c|}
\hline 51 & 55 & 59 & 63 \\
\hline 2 & 6 & 10 & 14 \\
\hline 17 & 21 & 25 & 29 \\
\hline 32 & 36 & 40 & 44 \\
\hline
\end{tabular}

\begin{tabular}{|c|c|c|c|}
\hline 3 & 7 & 11 & 15 \\
\hline 18 & 22 & 26 & 30 \\
\hline 33 & 37 & 41 & 45 \\
\hline 48 & 52 & 56 & 60 \\
\hline
\end{tabular}

Figure 4: Ordering of the 64 nibbles of the internal state after applying $\mathrm{SR}_{r}$ depending on $r \bmod 4$, when the cube is represented as the collection of its 4 slices. 


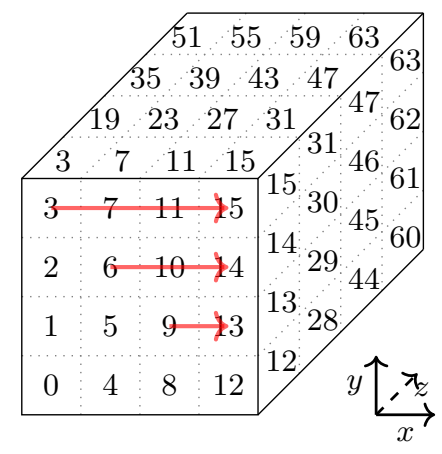

(a) $\mathrm{SR}_{\text {slice }}($ when $r \equiv 1 \bmod 4)$

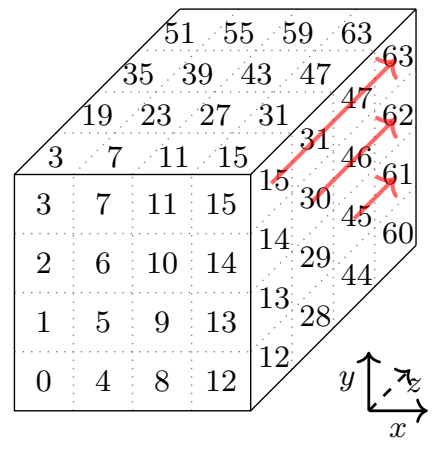

(b) $\mathrm{SR}_{\text {sheet }}($ when $r \equiv 3 \bmod 4)$

Figure 5: Representation of the $\mathrm{SR}_{r}$ operations on the cube. 


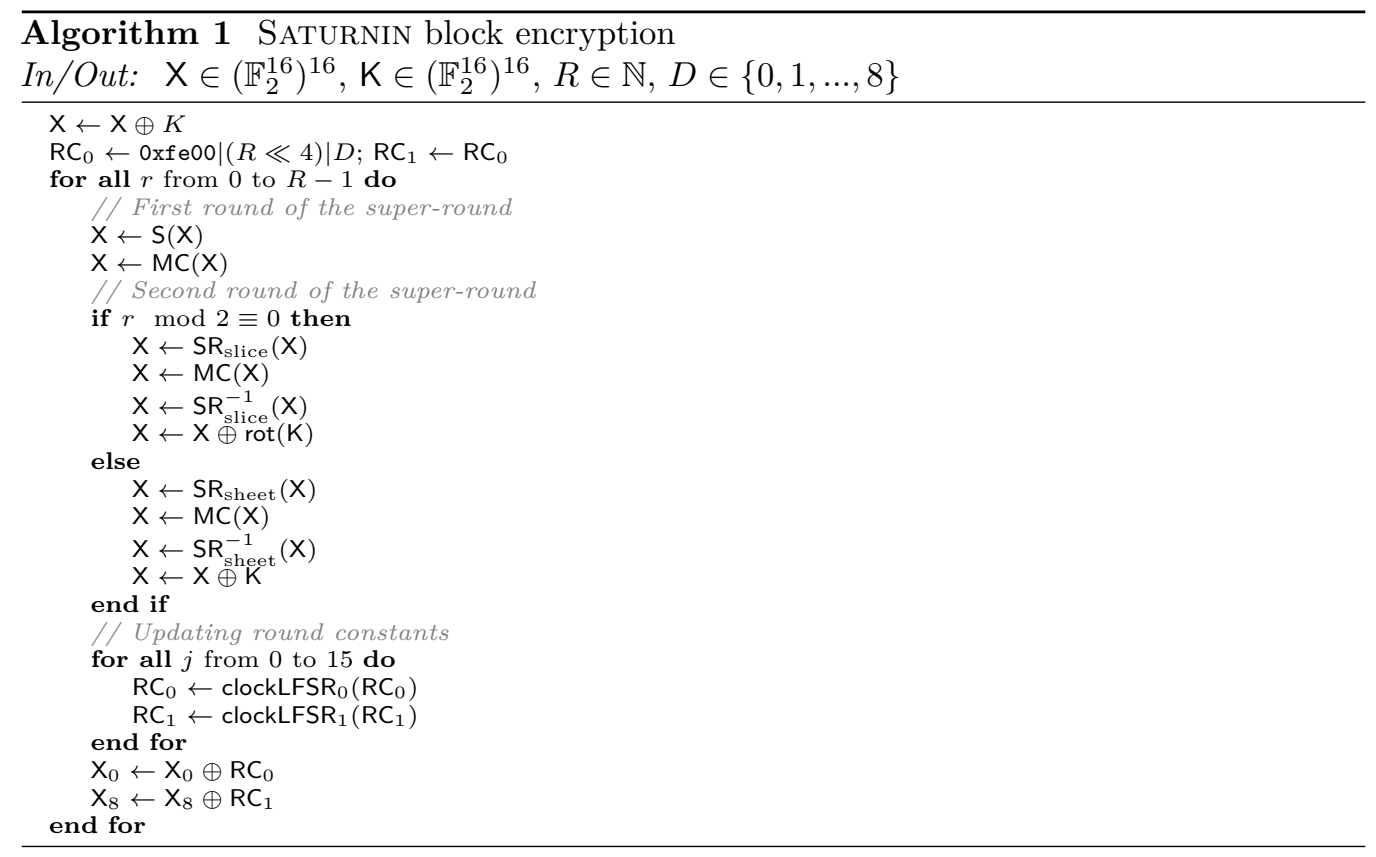

\subsection{The Authenticated Cipher Saturnin-CTR-Cascade}

We first propose to use the SATURNIN block cipher with modes known for their robustness against quantum adversaries: we combine the counter mode (Figure 6) for encryption and the Cascade construction [BCK96] for authentication, following the Encrypt-then-MAC composition [BN00]. The Cascade construction is used, for example, in NMAC [BCK96], and our MAC is very similar to NMAC based on SATURNIN in Matyas-Meyer-Oseas (MMO) mode [MMO85]. The nonce has length up to 160 bits and the tag has up to 256 bits (it can be truncated).

The nonce is first padded into the 161-bit string $N$ by appending to the nonce a bit of value 1 , then as many bits of value 0 as needed to reach a total length of 161 bits.

In general, whenever our proposed modes (SATURNIN-CTR-Cascade and SATURNINHash) require padding a value of less than 256 bits into a 256 -bit block, we use the following padding rule, and denote as $\operatorname{pad}(x)$ the padding of block $x$.

Padding rule: The padding rule consists in appending a single bit of value 1, followed by as many zeroes as necessary to reach the next block boundary.

In the counter (CTR) mode depicted on Figure 6, we define a keystream by encrypting blocks composed of $N$ concatenated with a 95-bit counter which is increased by one for each new call: $z_{i}=\operatorname{SATURNIN}^{1}(k, N \| i+1)$ (the counter starts at 1 for the block $\left.z_{0}\right)$. The counter is encoded with the big-endian convention: if the counter numerical value $u$ is:

$$
u=\sum_{i=0}^{94} u_{i} 2^{i}
$$

then each bit $u_{i}$ becomes bit $255-i$ in the input block. Thus, in a byte-oriented implementation and with a 128-bit nonce, the input to SATURNIN ${ }^{1}$ for the computation of $z_{0}$ will consist in the 16 bytes of the nonce, followed by a byte of value $0 \times 80$ (first padding byte for the nonce), followed by 14 bytes of value $0 \times 00$, followed by one byte of value $0 \times 01$. 
We split the input plaintext $m$ into full blocks $m_{0}, m_{1}, \ldots m_{\ell}$ and a final partial block $m_{*}$. Similarly, the associated data $a$ is split into $a_{0}, a_{1}, \ldots a_{j}, a_{*}$. The SATURnin-CTR-Cascade encryption process is then:

- Encryption:

1. For all $i=0$ to $\ell: c_{i} \leftarrow m_{i} \oplus \operatorname{SATURnin}^{1}(k, N \| i+1)$

2. $c_{*} \leftarrow m_{*} \oplus \operatorname{trunc}_{n}\left(\operatorname{SATURNIN}^{1}(k, N \| \ell+2)\right.$ ) (where trunc ${ }_{n}$ truncates its input to its first $n$ bits, $n$ being the length, in bits, of $\left.m_{*}\right)$.

- Authentication tag:

1. $t \leftarrow(N \| 0) \oplus \operatorname{SATURNIN}^{2}(k, N \| 0)$

2. For all $i=0$ to $j: t \leftarrow a_{i} \oplus \operatorname{SATURNIN}^{2}\left(t, a_{i}\right)$

3. $t \leftarrow \operatorname{pad}\left(a_{*}\right) \oplus \operatorname{SATURNIN}^{3}\left(t, \operatorname{pad}\left(a_{*}\right)\right)$

4. For all $i=0$ to $\ell: t \leftarrow c_{i} \oplus \operatorname{SATuRnin}^{4}\left(t, c_{i}\right)$

5. $t \leftarrow \operatorname{pad}\left(c_{*}\right) \oplus \operatorname{SATURNIN}^{5}\left(t, \operatorname{pad}\left(c_{*}\right)\right)$

The ciphertext is $c=c_{0}\left\|c_{1}\right\| \ldots\left\|c_{\ell}\right\| c_{*}$; it has the same length as the plaintext. The authentication tag is the last value of $t$. When decrypting, the authentication tag is first recomputed, and compared with the received value; on mismatch, the encrypted message is rejected. If the authentication tags match, then decryption is identical to encryption. The comparison between authentication tags should endeavour not to reveal the bit position at which mismatched tags diverge.

All SAturnin calls use $R=10$ super-rounds. Five domain values are used:

- SAturnin ${ }^{1}$ : for CTR encryption;

- SATURNin ${ }^{2}$ : for Cascade over the associated data full blocks;

- SAturnin ${ }^{3}$ : for Cascade over the padded last block of associated data;

- SAturnin ${ }^{4}$ : for Cascade over the ciphertext full blocks;

- SATURnin ${ }^{5}$ : for Cascade over the padded last block of ciphertext.

\subsection{The Authenticated Cipher Saturnin-Short}

For some practical usages, we propose a third authenticated cipher for handling messages of length strictly less than 128 bits (without additional data) and nonces of size up to 128 bits: SATURNIN-Short (Figure 9). We denote by $\|$ concatenation of bit-strings, and again adopt the same padding convention as in Section 2.2 for messages shorter than 128 bits.

In SATURNin-Short, we use the fact that SATURNin has a 256-bit block size, allowing to mix together the nonce and the message. We use the variant SATURNIN ${ }^{6}$. It is worth noticing that the ciphertext and the tag are not two separate values. Given a nonce $N$ and a ciphertext $c$, the tag can be verified by deciphering $c$ and comparing the left half with $N$.

Since SATURNIN-Short does not allow additional data, we recommend that protocols based on SATURNIN-Short use a counter as the nonce to prevent reordering of the ciphertexts and to detect replay attacks. 

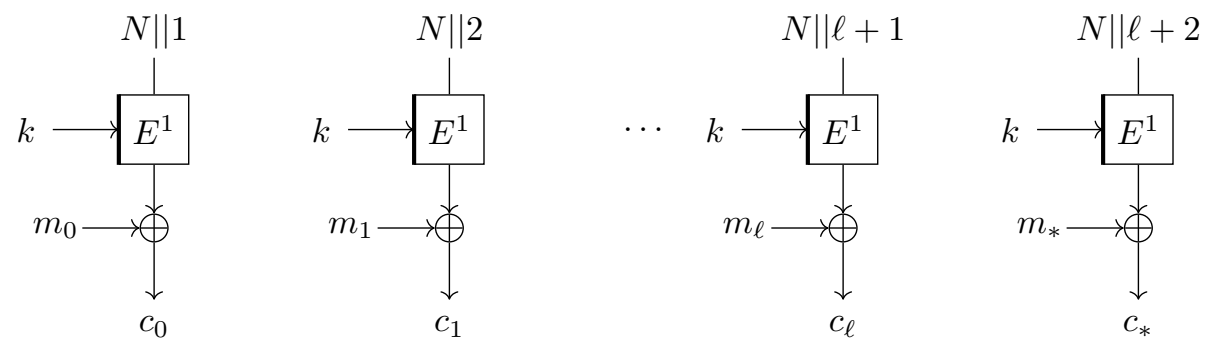

Figure 6: Counter Mode (CTR) encryption ( $E^{i}$ denotes a tweakable block cipher with Tweak $i$. A thick line represents the input of the key.

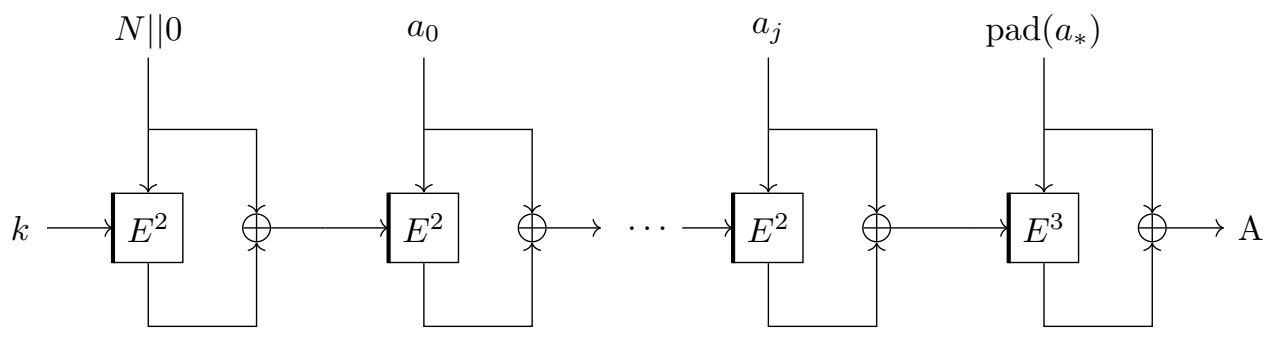

Figure 7: Cascade construction, processing of the associated data. A thick line represents the input of the key.

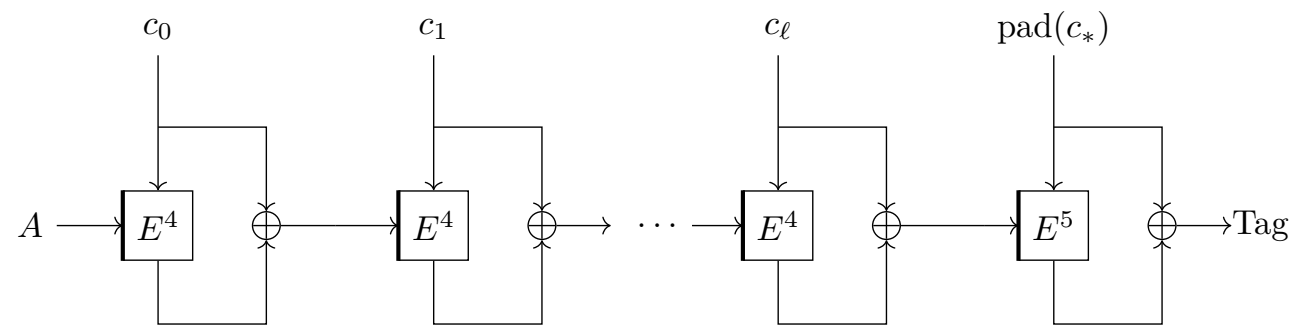

Figure 8: Cascade construction, processing of the ciphertext and computation of the tag. A thick line represents the input of the key.

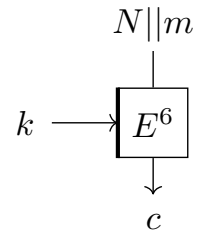

Figure 9: SATURNin-Short with key $k$, message $m$ and nonce $N$. A thick line represents the input of the key. 
Table 2: Correspondence between the value of the domain separator $D$ and the usage of SATURNIN $^{D}$

\begin{tabular}{|l|l|}
\hline Value of $D$ & Use \\
\hline \hline 0 & SATURNIN block cipher \\
1 & SATURNIN-CTR \\
2 & SATURNIN-Cascade AD \\
3 & SATURNIN-Cascade AD final \\
4 & SATURNIN-Cascade message \\
5 & SATURNIN-Cascade message final \\
6 & SATURNIN-Short \\
7 & SATURNIN-Hash \\
8 & SATURNIN-Hash final \\
\hline
\end{tabular}

\subsection{The Hash Function Saturnin-Hash}

We also propose a 256-bit hash function based on SATURNIN with the Merkle-Damgård construction [Mer90, Dam90], as shown in Figure 10. We use SATurnin 16 (i.e. SATurnin with 16 super-rounds) because we want the compression function to be resistant to relatedkey attacks. The compression function uses the MMO mode $\left(f\left(c_{i}, m\right)=E_{c_{i}}(m) \oplus m\right)$ to compress a 256-bit chaining value $c_{i}$ and a 256-bit message block $m$. Therefore, SATURNINhash is similar to the above Cascade, except that there is no key as starting point but a fixed value, 0 .

As in SATURnin-CTR-Cascade, the input message $m$ is split into full blocks $m_{0}, m_{1}, \ldots m_{\ell}$, and a final partial block $m_{*}$ (which may be empty, but is never full). Processing is then:

1. $t \leftarrow 0$ (the all-zero 256-bit block)

2. For all $i=0$ to $\ell: t \leftarrow m_{i} \oplus \operatorname{SATURNIN}_{16}^{7}\left(t, m_{i}\right)$

3. $t \leftarrow \operatorname{pad}\left(m_{*}\right) \oplus \operatorname{SATURNIN}_{16}^{8}\left(t, \operatorname{pad}\left(m_{*}\right)\right)$

The hash output is the final value of $t$.

\subsection{Values of the Domain Separator}

The values of the domain separator $D$ are used to describe the variant of SATURNIN that will be used in each role of each mode. We summarize the correspondence in Table 2 .

\section{Security Claims}

In general, we believe that, for each element in the SATURNIN suite, there is no attack significantly better than the generic attacks against the corresponding construction. In the following, the definition of attack obviously depends on the type of primitive. For a block cipher, it refers to distinguishing attacks in the SPRP-setting. For authenticated encryption, it refers to chosen-plaintext attacks breaking the confidentiality of the plaintext or the integrity of the ciphertext, as specified by the NIST submission requirements. We now formulate specific security claims.

Classically, the complexity of an attack is determined by the following quantities: $\mathcal{T}$ is the time complexity, expressed in units equivalent to the cost of one evaluation of the involved SATURNIN block cipher; $\mathcal{D}$ is the data complexity (encryption and verification queries), expressed as a number of 256-bit blocks, and $p$ is the success probability of an 
adversary. It is worth noticing that $\mathcal{T} \geq \mathcal{D}$ since the time for generating the data must be taken into account.

Quantumly, we adapt these definitions. Quantum computations are traditionally written using quantum circuits and the time complexity is given by the number of quantum gates in the circuit. For us, $\mathcal{T}$ is counted in units equivalent to the number of gates of one evaluation of SATURNIN implemented as a quantum circuit (see Appendix A for more details and definitions regarding quantum computing). Hence our security levels are independent of the cost of a quantum circuit for SATURNIN. $\mathcal{D}$ is the number of 256-bit blocks queried to a single superposition oracle. For example, for a mode of encryption with variable message-length, the adversary chooses the message-length she wants to query and queries the oracle in superposition over the messages. These oracle calls are interleaved with the quantum computations and each one requires as many time units as the required number of (quantum) secret-key computations of SATURNIN. For simplicity, we do not make hybrid classical-quantum security claims and consider that classical oracle calls cost the same as quantum ones. Indeed, on the one hand, classical queries are a particular type of quantum queries, so they should be at least easier to make. On the other hand, assuming the converse means that quantum queries are cheap, and this only strengthens our claims.

Block cipher. In the following claims, by default, SATURNIN denotes the block cipher with at least 10 super-rounds, i.e. 20 single-rounds as a super-round is formed by 2 rounds. When explicitly mentioned, SATURNIN 16 corresponds to the block cipher with 16 superrounds or more.

\section{Security Claim for SATURNIN block cipher (Section 2.1)}

There exists no classical attack in the single-key setting with $\mathcal{T} / p<2^{224}$. SATURNIN $_{16}$ provides a similar security level against related-key attacks involving a small number of keys ${ }^{a}$.

There exists no quantum attack in the single-key setting with $\mathcal{T}^{2} / p<2^{224}$. SATURNIN does not provide security against related-key superposition attacks (as is the case of all known block ciphers).

${ }^{a}$ with related-key deriving functions satisfying the conditions of [BK03].

Authenticated encryption. In the following security claims, $t$ is the length of the tags in bits ( $t$ is 256 by default, but can be less if the tags are truncated).

None of the AE schemes in SATURNIN provides security in nonce-misuse, noncerepetition or nonce-superposition scenarios ${ }^{3}$. In the single-key setting, we make the following claims:

\section{Security Claim for SATURnin-CTR-Cascade (Section 2.2)}

There exists no classical attack satisfying $\frac{\mathcal{D}^{2}+\mathcal{T}+\mathcal{D} 2^{256-t}}{p}<2^{224}$.

There exists no quantum attack satisfying $\frac{\mathcal{D}^{3}+\mathcal{T}^{2}+\mathcal{D}^{2} 2^{256-t}}{p}<2^{224}$.

\footnotetext{
${ }^{3}$ By nonce-superposition scenario we consider a quantum nonce-misuse scenario in which the adversary could call the encryption oracle in superposition over the nonces.
} 
In particular, in the case where $t=256$ these claims imply that any classical attack with high success probability must satisfy $\mathcal{D} \mathcal{T}>2^{224}$, which corresponds to the birthday bound at the 224-bit security level.

\section{Security Claim for SATURNIN-Short (Section 2.3)}

There exists no classical attack with $\frac{\mathcal{D}^{2}+\mathcal{T}+\mathcal{D} 2^{128}}{p}<2^{224}$.

There exists no quantum attack with $\frac{\mathcal{D}^{3}+\mathcal{T}^{2}+\mathcal{D}^{2} 2^{128}}{p}<2^{224}$.

The AE claims above are written in the single-key setting, assuming that related-key issues are dealt with at the protocol level. However, we also claim security against classical related-key attacks (with the same restrictions on the related keys as for the block cipher claims) when SATURNIN is replaced by SATURNIN 16 .

Hash function. The hash function in the SATURNIN suite is based on SATURNIN 16 . A lower number of super-rounds is not recommended. In what follows, $\mathcal{M}_{q}$ is the size of the quantum memory measured in registers of 256 qubits. In these claims we consider $p \geq 1 / 2$. We assume of course that $\mathcal{M}_{q} \geq 1$.

\section{Security Claim for SATURNIN-Hash (Section 2.4)}

There exists no classical collision attack with $\mathcal{T}<2^{112}$. There exists no classical second-preimage attack with $\mathcal{T}<2^{224-\ell}$ for messages of length $2^{\ell}$. There exists no classical preimage attack with $\mathcal{T}<2^{224}$.

There exists no quantum collision attack verifying $\mathcal{T}^{5} \times \mathcal{M}_{q}<2^{448}$. There exists no quantum second-preimage attack with $\mathcal{T}<2^{112-\ell / 2}$ for messages of length $2^{\ell}$. There exists no quantum preimage attack with $\mathcal{T}<2^{112}$.

In particular, the claim for quantum collision attack implies that there is no such attack with $\mathcal{T}<2^{75}$, because we necessarily have $\mathcal{M}_{q}<\mathcal{T}$.

\section{Summary of recommended parameters}

Our recommended variants of SATURNIN use 10 super-rounds for authenticated encryption, and 16 super-rounds for hashing.

\section{Primary member.}

AEAD: SATURnin-CTR-Cascade with 10 super-rounds

Hash: SATURNin-Hash with 16 super-rounds

The primary member combines modes with strong post-quantum security guarantees, no need for the block-cipher decryption and no patent claims we are aware of. As mentioned in the previous section, we claim security up to the birthday bound, in the single-key setting.

\section{Variant: optimized for small messages}

AE: SATURNin-Short with 10 super-rounds

Hash: SATURnin-Hash with 16 super-rounds 
This variant is a special construction dedicated to short messages of at most 128 bits, without associated data. The authentication security is slightly decreased, equivalent to truncating the tag to 128 bits.

In practice, if there is a need to encrypt both short messages and longer messages, we recommend to use SATURNIN-CTR-Cascade with a 128-bit tag for longer messages, with an extra ciphertext bit for domain separation.

\section{Implementation}

\subsection{Bitslice Representation and Conversions}

The "cube of nibbles" representation of SATURNIN blocks, keys and state values uses 64 nibbles with coordinates $(x, y, z)$ (all three coordinates range from 0 to 3 ). Each nibble contains four bits, numbered from 0 (least significant) to 3 (most significant).

The "bitsliced" representation [DGV94] splits the value into sixteen registers of 16 bits each. A bit within a register is indexed by values $(i, b)$, where $i$ is the register number $(0$ to 15$)$, and $b$ is the bit number (0 to 15). Within a register, bit 0 is least significant, and 15 is most significant. The register bits map to the nibble bits in the following way:

$$
(4 j+k, b) \longrightarrow(b \bmod 4, j,\lfloor b / 4\rfloor)_{k} \quad(0 \leq j \leq 3,0 \leq k \leq 3,0 \leq b \leq 15)
$$

i.e. the bits 0 to 3 of the nibble $(b \bmod 4, j,\lfloor b / 4\rfloor)$ are the bits $b$ of registers $4 j, 4 j+1$, $4 j+2$ and $4 j+3$, respectively.

The "bits" representation encodes the registers of the bitsliced representation into bits. The traditional mixed-endian convention is used: the two octets (bytes) of a register are encoded in little-endian order (least significant octet first), but bits within each octet are reputed to be ordered from most significant to least significant. Registers are encoded in ascending numerical order (0 to 15$)$.

In most practical implementations, SATURNIN will operate on keys and blocks which are already grouped into octets. In that case, this preexisting grouping of bits into octets is assumed to already follow the convention prescribed above. Therefore, the first two octets $t_{0}$ and $t_{1}$, each with numerical value 0 to 255 , encode register 0 with numerical value $t_{0}+256 t_{1}$; the next two octets $t_{2}$ and $t_{3}$ encode register 1 with numerical value $t_{2}+256 t_{3}$; and so on.

In a byte-oriented implementation, the ordering of bits within a byte matters only for the padding rule used in SATURNIN-Hash and the AEAD modes: when appending a bit of value 1 followed by zeros, this translates to appending a byte of value 0x80 followed by bytes of value $0 \times 00$.

It shall be noted that in SATURNIN-CTR-Cascade, we specified the encoding of the counter to use big-endian, since this is the standard encoding in other AEAD modes such as GCM, CCM or EAX. The conversion of the counter numerical values into the values of the high-index registers will thus imply some byteswapping.

\subsection{Re-interpreting the Operations of Saturnin}

While specified over a cube, SATURNIN is best implemented using a bitsliced approach. For implementation purposes, the internal state of SATURNIN is represented as sixteen 16-bit registers indexed from 0 to 15 (Figure 1b). In this register representation, the bit of lowest weight of each register has index 0 and the bit of highest weight has index 15. A bit is then defined by the index $i$ of its register and its index $b$ within its register. The correspondence between the register representation and the cube representation is as follows: the bits with coordinates $(4 i, b),(4 i+1, b),(4 i+2, b)$ and $(4 i+3, b)$, which are therefore taken from registers $4 i$ to $4 i+3$, correspond to nibble $(b \bmod 4, i,\lfloor b / 4\rfloor)$ in the cube (see Figure 1$)$. 
Table 3: The correspondence between the input and output bit indices in $\pi_{0}$ and $\pi_{1}$.

\begin{tabular}{ccccc}
\hline$i$ & 0 & 1 & 2 & 3 \\
\hline$\pi_{0}(i)$ & 3 & 0 & 1 & 2 \\
$\pi_{1}(i)$ & 2 & 1 & 3 & 0 \\
\hline
\end{tabular}

A slice in the cube corresponds to bits with indices $(i, b)$ such that $\lfloor b / 4\rfloor$ is constant, while a sheet corresponds to bits with indices $(i, b)$ such that $(b \bmod 4)$ is constant. Therefore, the columns in the cube are composed of bits $(i, b)$ with a constant $b$ in the registers.

The transformations involved in the round function of SATURNIN are then implemented as follows.

S-box Layer. The 4-bit S-boxes $\sigma_{0}$ and $\sigma_{1}$ are applied in parallel, each over a half of the whole state. These permutations are such that $\sigma_{0}=\pi_{0} \circ \sigma$ and $\sigma_{1}=\pi_{1} \circ \sigma$ where $\pi_{0}$ and $\pi_{1}$ are bit permutations. Their action on the index of the bits in each nibble is given in Table 3.

In the register representation, the S-boxes are applied in a bitsliced fashion over all registers with indices $4 i+0, \ldots, 4 i+3$, where $i \in\{0,2\}$ for $\sigma_{0}$ and $i \in\{1,3\}$ for $\sigma_{1}$. The full parallel application of the S-boxes is denoted $\mathrm{S}$ and, in the register representation, it is summarized in Figure 11.

The lookup-tables of the S-boxes are given in Section 2.1.2. They can be implemented in bitslice over 4 words using the $\mathrm{C}$ macros in Figure $11 \mathrm{~b}$.

MDS Matrix. The matrix $M$ is defined over $\left(\mathbb{F}_{2^{4}}\right)^{4}$ and is applied in parallel on each column of the state. In the register representation, it is applied in a bitsliced fashion over the whole state at once as summarized in Figure 12a.

The matrix is one of the low-cost MDS matrices found in [DL18]. It can be implemented in a bitsliced fashion using the $\mathrm{C}$ macro in Figure $12 \mathrm{~b}$.

$\mathbf{S R}_{\text {slice. }}$ In order to mix the columns in each slice, we use $\mathrm{SR}_{\text {slice }}$ which consists in the parallel application of $R_{\text {slice }}$ on each slice independently. This operation maps the nibble with coordinates $(x, y, z)$ to $(x+y \bmod 4, y, z)$. Its implementation on 16-bit registers is summarized in Figure 13.

It can be implemented by applying to register $i$ a rotation of each 4-bit word in it by $\lfloor i / 4\rfloor$. The following $\mathrm{C}$ macros implement the 4 such functions we need:

- the rotation by 1 is $((x \& 0 x 7777)<<1) \mid((x \& 0 x 8888)>>3)$,

- the rotation by 2 is $((x \& 0 x 3333)<<2) \mid((x \& 0 x c c c c)>>2)$, and

- the rotation by 3 is $((x \& 0 x 1111)<3) \mid((x \&$ oxeeee $)>>1)$.

ShiftRows in a Sheet. We proceed with sheets as we did with slices. In order to mix the columns in each sheet, we use $\mathrm{SR}_{\text {sheet }}$ which consists in the parallel application of $R_{\text {sheet }}$ on each sheet independently. This operation maps the nibble with coordinates $(x, y, z)$ to $(x, y, z+y \bmod 4)$. It is summarized in Figure 14. It can be implemented with a rotation of the 16-bit register $i$ by $4\lfloor i / 4\rfloor$. 


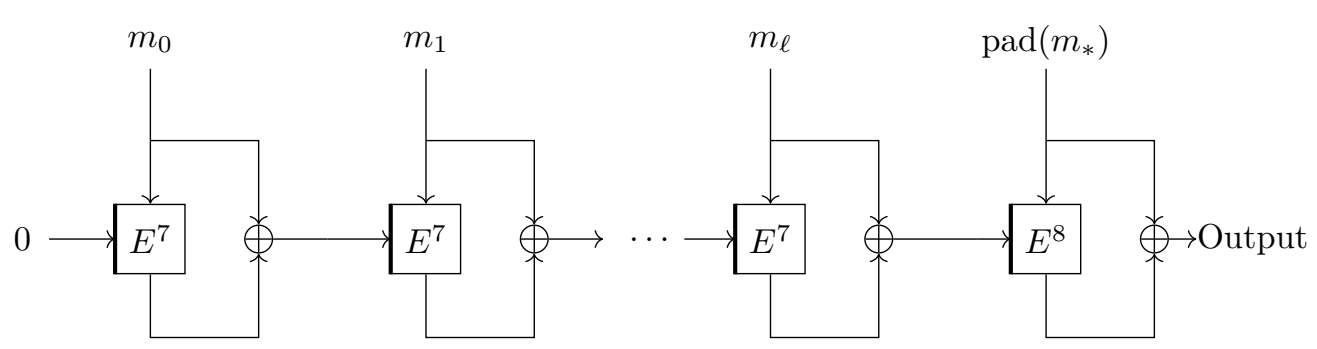

Figure 10: The hash function SATURNIN-Hash, where $E^{i}$ is SATURNIN 16 with domain separator $i$. A thick line represents the input of the key.

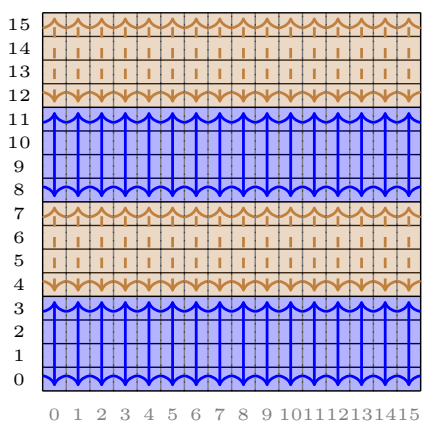

(a) As applied in the registers.

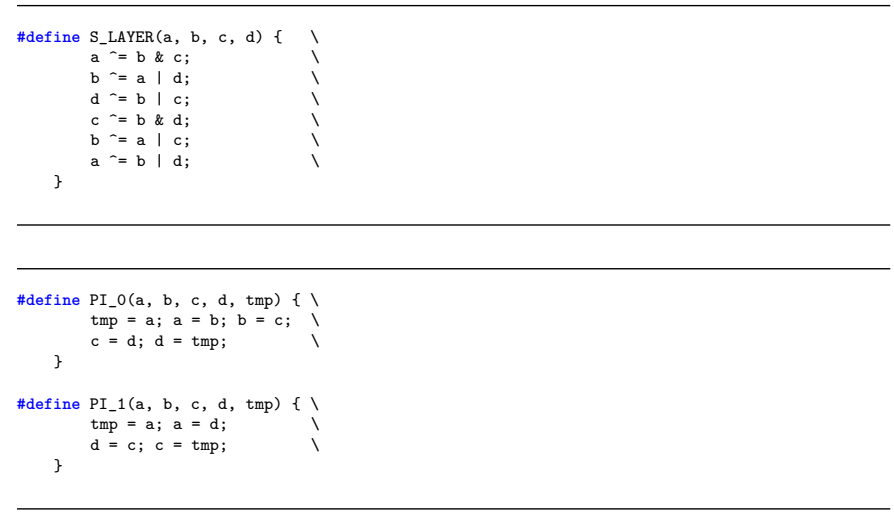

(b) The main S-box $\sigma$ and the bit permutations $\pi_{0}$ and $\pi_{1}$.

Figure 11: The application of the 4-bit S-boxes $(\mathrm{S})$ in the register representation. $\sigma_{0}$ is represented by continuous blue arrows, $\sigma_{1}$ by dashed brown ones.

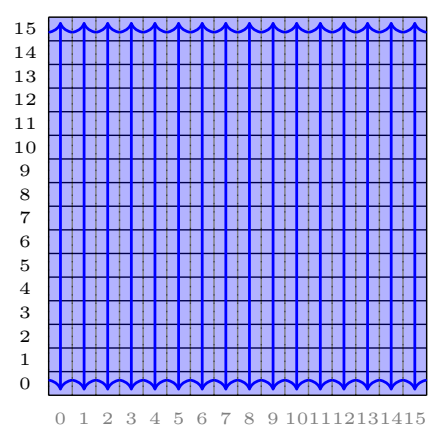

(a) In register representation.

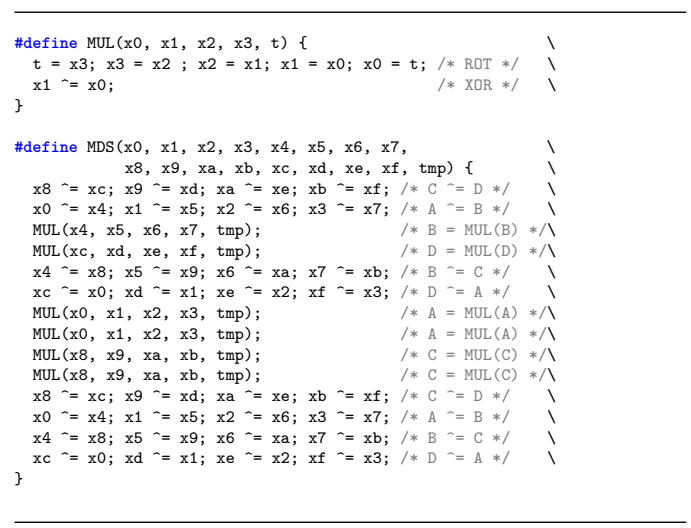

(b) As a C macro.

Figure 12: The parallel application of the $16 \times 16$ matrix $M$, i.e. the operation $M C$. 


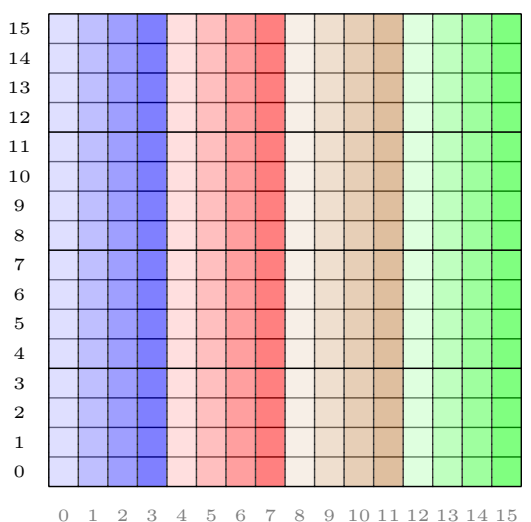

(a) Before.

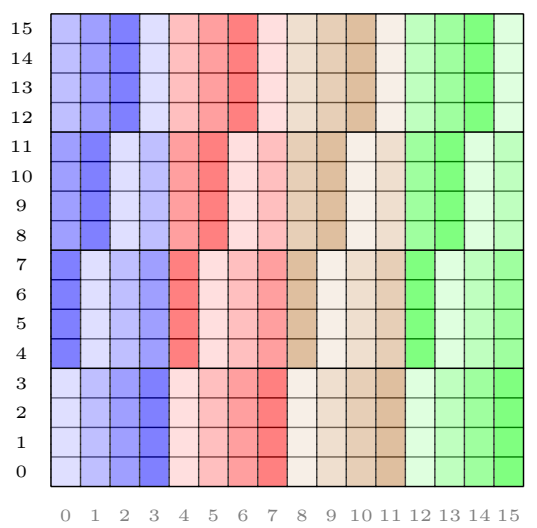

(b) After.

Figure 13: The $\mathrm{SR}_{\text {slice }}$ operation that mixes the columns in each slice separately.

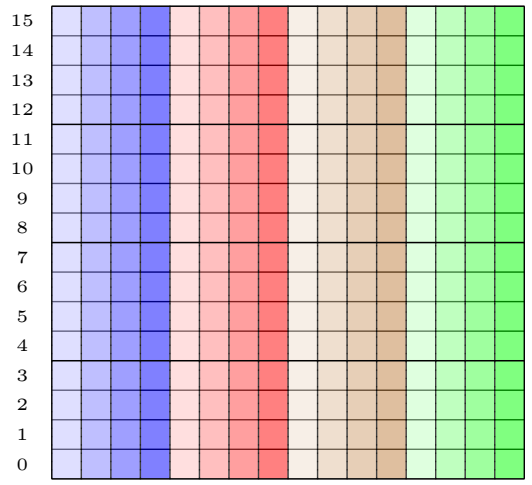

(a) Before.

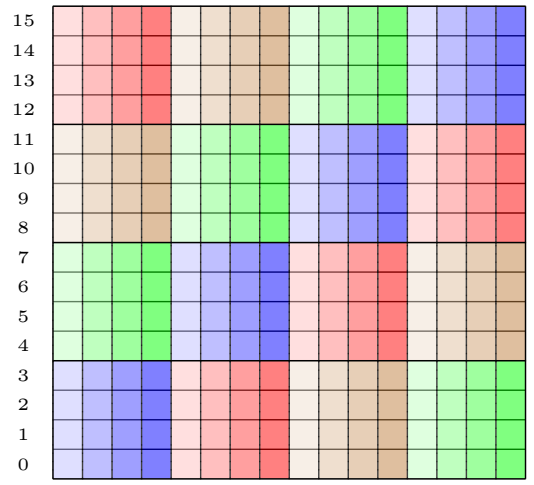

$\begin{array}{lllllllllll}0 & 1 & 2 & 3 & 4 & 5 & 6 & 7 & 8 & 9 & 101112131415\end{array}$

(b) After.

Figure 14: The $\mathrm{SR}_{\text {sheet }}$ operation that mixes the columns in each sheet separately. 
\#define CLOCK_LFSR_O $(\mathrm{x}) \quad \mathrm{x}=((\mathrm{x}) \& 0 \mathrm{x} 8000) ?((\mathrm{x}) \ll<1)-0 \mathrm{x} 002 \mathrm{~d}:(\mathrm{x})<1$;

\#define CLOCK_LFSR_1 $(\mathrm{x}) \quad \mathrm{x}=((\mathrm{x}) \& 0 \mathrm{x} 8000) ?((\mathrm{x}) \ll 1)-0 \mathrm{x} 0053:(\mathrm{x})<1$;

Figure 15: Clocking the LFSRs used to derive the round constants.

Key Addition. The key addition is applied in a very straightforward way by XORing 16-bit registers in the key state with their counterparts in the internal state of the cipher. In odd super-rounds, each register of the master key is rotated just before being added, meaning we can use operators combining the XOR and the rotation when available.

Constant Addition. The bits in which the constants $\mathrm{RC}_{0}$ and $\mathrm{RC}_{1}$ are $\mathrm{XORed}$ correspond to registers 0 and 8 respectively. Hence, this operation is implemented using two word-wise XORs. The 16-bit state of each LFSR naturally corresponds to one 16-bit register, and one clock of each LFSR is easily implemented using the C macros in Figure 15.

\subsection{Operations Count}

In order to evaluate the efficiency of SATURNIN, we can count the number of operations required to encrypt one 256-bit block of plaintext. If we assume an ideal 16-bit instruction set with three-operand instructions, we need the following instructions for one round of SATURNIN:

- $4 \times 12$ instructions for the S-box layer (6 AND/OR, and 6 XOR)

- 38 XOR instructions for the MDS layer

- depending on the round number, either 12 rotation instructions for $\mathrm{SR}_{\text {sheet }}$ or $\mathrm{SR}_{\text {sheet }}^{-1}$, or 60 instructions (two ANDs for masking, two shifts, and one OR for each of the 12 registers) for $\mathrm{SR}_{\text {slice }}$ or $\mathrm{SR}_{\text {slice }}^{-1}$.

- 16 XOR instructions every two rounds for the key addition. ${ }^{4}$

In total, with 20 rounds plus the last key addition (and ignoring the key schedule), this makes 2616 instructions to process 256 bits, or 10.2 instructions per bit.

In a hardware setting, the nibble permutations $\mathrm{SR}$ and $\mathrm{SR}^{-1}$ are free, ${ }^{5}$ and we just need the following operations:

- $64 \times 12$ gates for the S-box layer $(6 \mathrm{AND} / \mathrm{OR}$, and $6 \mathrm{XOR})$

- $16 \times 38$ XOR gates for the MDS layer

- 256 XOR gates every two rounds for the key addition

In total, with 20 rounds, this sums up to 30336 gates to process 256 bits, or 118.5 gates per bit. For reference, we can compare this number with the results for SKINNY [BJK $\left.{ }^{+} 16\right]$, SIMON [BSS $\left.{ }^{+} 13\right]$, NOEKEON [DPVAR00] and AES that are given in [BJK $\left.{ }^{+} 16\right]$, even though those ciphers have a smaller block size of 128 bits. If we ignore the cost of the key schedule, at the 128-bit security level, SKINNY requires 130 gates per bit, SIMON requires 136, NOEKEON 100, and AES 202.5. Focusing on ciphers with a 256-bit key, we need 156 operations for SKINNY, 144 for for SIMON, and 283.5 for AES. This shows that SATURNIN is a good candidate for efficient implementations.

\footnotetext{
${ }^{4}$ To which two XORs must be added if the subkeys are computed in the fly along with the instructions implementing the LFSRs updating the round constants.

${ }^{5}$ This is true for an implementation unrolling 4 rounds (2 super-rounds). An implementation performing fewer rounds per clock cycle will need additional multiplexers to take the differences between our operations into account.
} 


\subsection{Software Implementations}

In the bitslice representation, the state and keys of SATURNIN are naturally expressed as sequences of 16-bit registers. However, many small microcontrollers now offer a 32-bit architecture, in particular the ARM Cortex-M line. Bigger architectures may also offer larger registers. There are several possible strategies for representing the state of one or several instances of SATURNIN. We implemented in $\mathrm{C}$ three strategies called bs32, bs32x and bs64.

In bs32, the sixteen 16-bit registers of SATURNIN $\left(r_{0}\right.$ to $\left.r_{15}\right)$ are stored in eight 32-bit registers $q_{0}$ to $q_{7}$, such that the 16 low bits of $q_{i}$ contain $r_{i}$, and the 16 high bits of $q_{i}$ contain $r_{i+8}$. This representation allows sharing some operations that are performed identically on $r_{i}$ and $r_{i+8}$, such as $\sigma_{0}$ (applied on $r_{0}$ to $r_{3}$, and on $r_{8}$ to $r_{11}$ ) and $\sigma_{1}$, the multiplications by $\alpha$ in the linear layer MC, and addition of round constants.

In bs32x, two independent SATURNIN instances are executed in parallel as described in $\left[\mathrm{BDP}^{+} 12\right]$ in the case of Keccak. If we call $\left(r_{i}\right)$ the sixteen 16-bit state registers of the first instance, and $\left(s_{i}\right)$ the sixteen 16-bit state registers of the second instance, then we use sixteen 32-bit registers $\left(w_{i}\right)$ such that each $w_{i}$ contains the interleaved bits of $r_{i}$ and of $s_{i}$ : bit $j$ of $r_{i}$ is stored in bit $2 j$ of $w_{i}$, and bit $j$ of $s_{i}$ is stored in bit $2 j+1$ of $w_{i}$. This representation allows parallel evaluation of the $\mathrm{S}$-box and linear layers, and, compared to bs32, simplifies data movements. However, it requires some extra operations for interleaving the state values on input (and the reverse operation on output), uses twice as many registers, and is applicable only in cases where parallel evaluation of two SATURNIN instances is possible (e.g. in SATURNin-CTR-Cascade, but not in SATURnin-Hash).

The bs64 representation is an extension of bs32 for 64-bit architectures: it uses eight 64-bit registers, whose low halves contain the $q_{0}$ to $q_{7}$ values of bs32 for one SATURNIN instance, and whose high halves contain the $q_{0}$ to $q_{7}$ of another independent instance. This strategy allows sharing the computations of bitwise operations (e.g. $\sigma_{0}$ and $\sigma_{1}$ ), but requires more operations for the linear layer and the SR operations. Moreover, like bs32x, it requires two SATURNIN instances to evaluate in parallel.

In our tests, bs32x offers a slight performance increase, compared to bs32, on registerrich architectures (e.g. PowerPC) but not on register-starved CPU (e.g. x86 and low-end ARM). On 32-bit architectures, bs64 has no advantage over bs32, but even on 64-bit systems, the bs64 implementation is only marginally faster than bs32, even in optimal conditions.

Therefore, bs32 seems to be the best performing, and most flexible, on low-end 32-bit systems of the ARM Cortex line. We reimplemented it in hand-optimized assembly for the ARM Cortex-M3 and ARM Cortex-M4; the M4 implementation leverages some opcodes (pkhbt and pkhtb) that the M3 does not feature, making that code slightly smaller and faster. Figure 16 shows the resulting code footprint (in bytes) and data throughput (in cycles per byte, for hashing, encrypting, and processing the Additional Authenticated Data (AAD)). Three implementations (portable C, assembly for Cortex-M4, assembly for Cortext-M3) are compared. All three implementations follow a common, realistic, streamable API amenable to integration in various applications.

\section{Rationale}

\subsection{General Structure using the Super-S-box Representation}

The main motivation behind the general structure of SATURNIN is to mimic the structure of the AES, which is arguably the best understood symmetric primitive. Indeed, the Super-S-box view of SATURNIN is very similar to an AES operating on 16-bit words, instead of bytes. Let us denote by $\mathrm{S}_{16}$ the 16-bit Super-S-box in SATURnin, i.e., the permutation of $\mathbb{F}_{2}^{16}$, composed of the succession of an S-box layer, the linear MDS function $M$, and a 


\begin{tabular}{|l|r|rrr|}
\hline \multirow{2}{*}{ Implementation } & \multirow{2}{*}{ Code size } & \multicolumn{3}{|c|}{ Throughput (c/B) } \\
& & Hash & Encrypt & AAD \\
\hline saturnin_portable.c & 3956 & 183 & 250 & 128 \\
saturnin_m4.s & 2948 & 111 & 144 & 75 \\
saturnin_m3.s & 3028 & 113 & 147 & 77 \\
\hline
\end{tabular}

Figure 16: Size and performance of streamable implementations of SATURNIN-CTRCascade and SATURnin-Hash on ARM Cortex-M4. The code size is expressed in bytes; the processing speeds for hashing, encryption, and additional authenticated data, are given in cycles-per-byte.

second S-box layer. The composition of two rounds of indices $(2 t, 2 t+1)$ can then be seen as the application of this Super-S-box to each column of the internal state, followed by $\mathrm{SR}_{2 t+1}^{-1} \circ \mathrm{MC} \circ \mathrm{SR}_{2 t+1}$. If $t$ is even, then the slices are invariant under $\mathrm{SR}_{2 t+1}$. This implies that the linear layer $\mathrm{SR}_{2 t+1}^{-1} \circ \mathrm{MC} \circ \mathrm{SR}_{2 t+1}$ consists of the concatenation of four copies of the same function $\mathrm{L}_{64}$ of $\left(\mathbb{F}_{2}^{16}\right)^{4}$, which applies to the slices independently.

If $t$ is odd, then the sheets are invariant under $\mathrm{SR}_{2 t+1}$. In this case, the linear layer consists of the concatenation of four copies of the same function $\mathrm{L}_{64}$, which applies to the sheets independently. Moreover, it is easy to prove, e.g. by Theorem 1 in $\left[\mathrm{ADK}^{+} 14\right]$ that $\mathrm{L}_{64}$ has branch number 5 with respect to $\mathbb{F}_{2}^{16}$.

Let us then represent in a $4 \times 4$ matrix $C$ the 16 -bit words $C_{0,0}, \ldots, C_{3,3}$ corresponding to the 16 columns of the cube, where $\mathrm{C}_{i, j}$ corresponds to the column defined by $x=i, z=j$. This means that each slice in the cube is a column of Matrix C, while each sheet in the cube is a row of $\mathrm{C}$. When $t$ is even, the linear function $\mathrm{L}_{64}$ then applies to the columns of $\mathrm{C}$ independently, while for odd $t$, it applies to its rows. In other words, a super-round has the following structure: the S-box $\mathrm{S}_{16}$ is applied to each 16-bit word, then $\mathrm{L}_{64}$ is applied to the four columns of $\mathrm{C}$, and Matrix $\mathrm{C}$ is transposed.

SATURNIN is then very similar to an AES operating on 16-bit words, except that the ShiftRows transformation is here replaced by a transposition exactly as it was in SQUARE, a predecessor of the AES [DKR97]. In the following, this super-S-box view and the previous notation will be used for analyzing the resistance of SATURNIN to the main classes of attacks.

\subsection{On the Building-blocks in the Block Cipher}

\subsubsection{On the Number of Rounds}

The number of rounds has been determined by the security analysis, which shows that, for most attacks, a super-round in SATURNIN offers a resistance similar to a single round in the AES. Therefore, 10 super-rounds, i.e. 20 rounds, appears to be a natural choice.

\subsubsection{On the MDS Matrix $M$}

The main building-block in the linear layer is the $4 \times 4$-MDS matrix $M$ over $\mathbb{F}_{2^{4}}$. This matrix is one of the MDS matrices exhibited in [DL18] with the lowest known implementation cost. Its Feistel-like structure guarantees that its inverse also has a low implementation cost.

Moreover, this MDS matrix applies similar operations to $a$ and $c$ (and to $b$ and $d$ ), see Figure 3. This allows an efficient 32-bit implementation, where the 16 bits corresponding to $a$ and the 16 bits corresponding to $c$ are stored in the same register (respectively the 16 bits corresponding to $b$ and the 16 bits corresponding to $d$ ). 


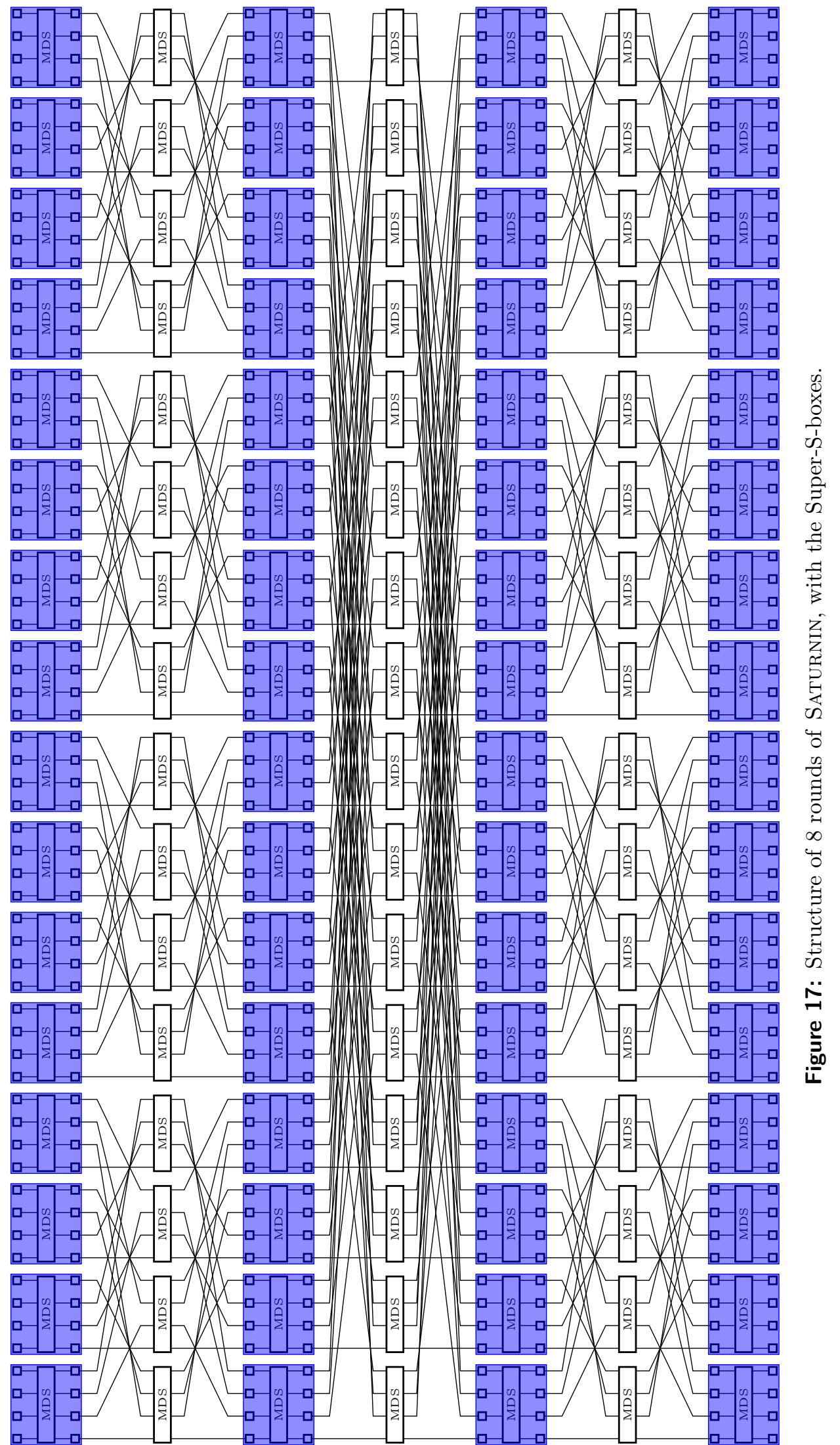




\subsubsection{On the Design of the Super-S-box and the Choice of $\sigma$}

The two S-boxes $\sigma_{0}$ and $\sigma_{1}$, which apply to the nibbles with an even index and with an odd index respectively, are both chosen as the composition of the same S-box, $\sigma$, followed by two different permutations of the output bits (see Table 3).

Choice of $\sigma$. The 4-bit S-box $\sigma$ has been chosen among the 4-bit S-boxes with optimal cryptographic parameters, i.e., in one of the equivalence classes named optimal in the classification established by Leander and Poschmann [LP07]. These S-boxes are those with differential uniformity 4 and linearity 8 (i.e. both their MDP and MLP are equal to $2^{-2}$ ). Among these S-boxes, we only considered those having all nonzero linear combinations of their coordinates of degree 3. Moreover, we chose for $\sigma$ an S-box minimizing the number of operations in the bitslice implementation, both for $\sigma$ and $\sigma^{-1}$ in order to compute efficiently the inverse cipher for decryption with SATURNIN-Short. We used a strategy inspired from $\left[\mathrm{UDI}^{+} 11\right]$ and searched, among all S-boxes with the lowest possible implementation cost, for those having the required cryptographic properties. It is worth noticing that the list of low-cost S-boxes computed by Ullrich et al. could not be used in our case. Indeed, since it is restricted to all S-boxes which could be implemented with at most 13 instructions, it does not contain any S-box having all its components of degree 3 . Also, we need to minimize the implementation cost of both the S-box and its inverse, which makes the approach different from $\left[\mathrm{UDI}^{+} 11\right]$. Instead, we chose to search for S-boxes with a Feistel-like structure, which can be easily inverted. The best such S-box we could find requires 6 XOR, 6 nonlinear instructions, corresponding to a total of 12 instructions (with three operands). Its inverse has a similar implementation cost.

Design of $\mathbf{S}_{\mathbf{1 6}}$. A counterpart of the nice implementation properties of the MDS matrix $M$ is that it transforms the subspace of $\mathbb{F}_{2^{4}}$ defined by $\left\{(x, x, 0,0), x \in \mathbb{F}_{2^{4}}\right\}$ into the subspace $\left\{(y, y, 0, y), y \in \mathbb{F}_{2^{4}}\right\}$. This implies that, if the nonlinear layer in $\mathrm{S}_{16}$ uses four copies of the same S-box $\sigma$, then $\mathrm{S}_{16}$ transforms the affine subspace of dimension 4 $\left\{\left(x, x, \sigma^{-1}(0), \sigma^{-1}(0)\right), x \in \mathbb{F}_{2^{4}}\right\}$ into the affine subspace $\left\{(y, y, \sigma(0), y), y \in \mathbb{F}_{2^{4}}\right\}$. We considered the propagation of such a 4-dimensional subspace, of a very simple form, as an unsuitable property. Moreover, this particular structure also explains why the Super-S-box $\mathrm{S}_{16}$ based on $\sigma$ only has a linearity equal to $2^{12}$ which is higher than expected. For these two reasons, we decided to use two slightly different S-boxes, one applied to the nibbles with an even index, and the other one applied to the nibbles with an odd index. We made an exhaustive search over all pairs of bit permutations $\left(\pi_{0}, \pi_{1}\right)$ of the four output bits, and studied the Super-S-box $\mathrm{S}_{16}$ derived from $\pi_{0} \circ \sigma$ and $\pi_{1} \circ \sigma$. The smallest differential uniformity that is obtained for these Super-S-boxes is equal to 80, and the smallest linearity (i.e., the highest magnitude of the Walsh transform) is equal to 3072. They are achieved simultaneously for two pairs $\left(\pi_{0}, \pi_{1}\right)$. Among these two possibilities, we chose the one leading to the Super-S-box with the lowest number of short cycles: most notably, for our choice, $\mathrm{S}_{16}$ has one fixed point (the all-zero word) and two cycles of length 6 , while for the other choice, $\mathrm{S}_{16}$ would have one fixed point, two cycles of length 2 , one cycle of length 3 , three cycles of length 4 , one cycle of length 5 and two cycles of length 6 . This motivated the choice of permutations $\pi_{0}$ and $\pi_{1}$ defined in Table 3: if $\sigma\left(x_{0}, x_{1}, x_{2}, x_{3}\right)=\left(y_{0}, y_{1}, y_{2}, y_{3}\right)$, then $\sigma_{0}\left(x_{0}, x_{1}, x_{2}, x_{3}\right)=\left(y_{1}, y_{2}, y_{3}, y_{0}\right)$ and $\sigma_{1}\left(x_{0}, x_{1}, x_{2}, x_{3}\right)=\left(y_{3}, y_{1}, y_{0}, y_{2}\right)$.

\subsubsection{On the Key Schedule and Constant Addition}

Since the key is aligned with the 16-bit structure of the cipher, we can reuse the analysis done on the AES and its variants to evaluate the security against related-key attacks (see e.g. $\left.\left[\mathrm{CHP}^{+} 17\right]\right)$. In particular, using a simple MILP model, we get the following lower 
bounds on the number of active Super-S-boxes in a related-key differential trail:

\begin{tabular}{lcccccccccc}
\hline$n$ (super-rounds) & 1 & 2 & 3 & 4 & 5 & 6 & 7 & 8 & 9 & 10 \\
\hline Active Super-S-boxes & 0 & 1 & 5 & 10 & 12 & 16 & 18 & 22 & 24 & 28 \\
\hline
\end{tabular}

Note that these are just lower bounds for truncated trails. In practice, many paths could be impossible to instantiate with concrete differences; indeed in the truncated model, two differences can always cancel out, but in a real trail, the constraints can be incompatible. These bounds are slightly better than the bounds given by the AES key schedule, and are the motivation behind our offset choice (other offsets gave fewer active S-boxes).

An example of iterative trail with 6 active Super-S-boxes every two super-rounds is given below, with MR denoting a super-round mixing inside the sheets (i.e. the rows in the matrix representation) and MC a super-round mixing inside the slices (i.e. the columns in the matrix representation), and $A K$ and $A K^{\prime}$ the key additions with the following key differences:
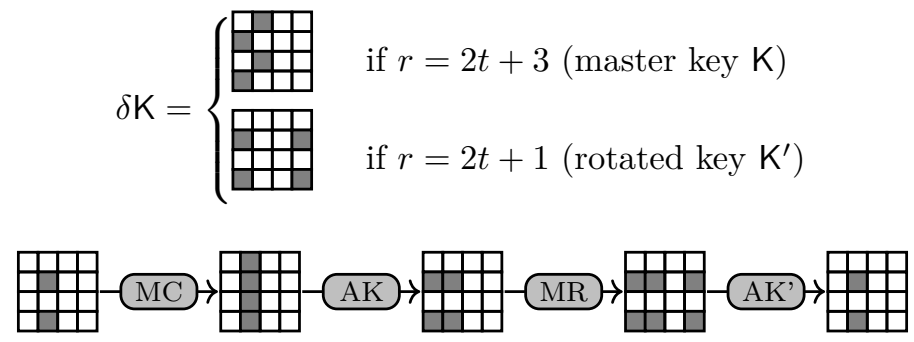

The first and last super-rounds can be optimized to remove one active Super-S-box, for instance, with 4 super-rounds:

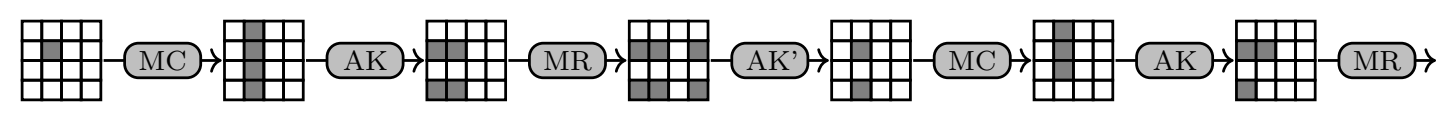

Therefore, this gives a trail with $3 r-2$ active Super-S-boxes for $r$ super-rounds (with $r$ even), which reaches the bound given above (in this example, we have $1+4+2+3$ active Super-S-boxes).

This is close to optimal for a linear key schedule, because there are simple trails where the full key is active with 7 active Super-S-boxes every two super-rounds, such as:

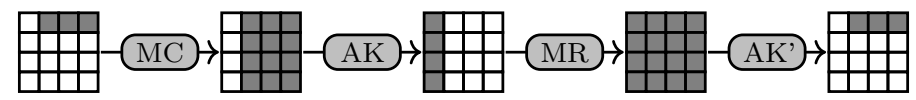

Round Constants. The sequence of round constants is generated by two 16-bit LFSRs run in parallel from the same seed. This aims at an efficient hardware implementation, while the whole sequence can be stored in software environments. Both feedback polynomials are primitive polynomials of degree 16 with the smallest number of monomials. They correspond to the first pair of primitive polynomials with 5 monomials in lexicographical order satisfying the following property: let us denote by $\left(x_{t}(b)\right)_{0 \leq t<512}\left(\operatorname{resp} .\left(y_{t}(b)\right)_{0 \leq t<512}\right)$ the binary sequence formed by the concatenation of the successive values of $\mathrm{RC}_{0}$ (resp. $\mathrm{RC}_{1}$ ) from the seed defined by the 9-bit integer $b$ (corresponding to the concatenation of the domain separator and the number of super-rounds). Then, in the round-constant sequences generated from the $2^{9}$ seeds, all sets of 16 consecutive pairs $\left(x_{t}, y_{t}\right)$ are distinct. In other words, there is no pair of distinct 9-bit integers $b$ and $b^{\prime}$ for which there exists $0<t_{0}<496$ such that $x_{t}(b)=x_{t+t_{0}}\left(b^{\prime}\right)$ and $y_{t}(b)=y_{t+t_{0}}\left(b^{\prime}\right)$ for all $t \geq 0$. 


\subsection{On Modes of Operation}

The different modes proposed, SATURnin-CTR-Cascade, SATURnin-Short and SATURninHash, are intended to provide quantum security against chosen plaintext and ciphertext superposition attacks (IND-qCPA and IND-qCCA), in addition to classical and quantum security against chosen message attacks and verification queries (IND-CCA). For authenticated encryption, we do not claim security in nonce-misuse scenarios or against the even stronger model of nonce-superposition attacks.

\subsubsection{Generic composition.}

One of the most natural ways to design an authenticated encryption scheme is to combine an encryption scheme and a MAC. The seminal work of Bellare and Namprempre [BN08] studied several composition methods in the classical setting: MAC-then-encrypt (used in TLS 1.0), encrypt-and-MAC (as used in SSH), and encrypt-then-MAC (used in IPSec). They showed that in the classical context, the encrypt-then-MAC construction offers the strongest security: it is generically secure against chosen-plaintext and chosen-ciphertext attacks (IND-CCA) assuming that the encryption scheme is secure against chosen-plaintext attacks (IND-CPA), and the MAC is unforgeable (SUF-CMA). In addition, the encryptthen-MAC composition allows to reject forgeries quickly without even decrypting the ciphertext. This also reduces the risk of inadvertently releasing an unverified plaintext.

In the quantum setting, Soukharev, Jao and Seshadri have revisited these results [SJS16], and proved that the encrypt-then-MAC composition offers IND-qCCA security under quantum security assumptions on the MAC and the encryption scheme. Their assumption on the MAC is SUF-qCMA, which is an adaptation of [BZ13a]. The encryption scheme needs IND-qCPA security. Both will be defined below.

\subsubsection{Quantum Security Definitions}

In this section, we formalize our quantum attacker models. In addition to classical chosen-message and verification queries, the attacker is allowed to make chosen-message superposition queries to the mode of encryption $E$, the MAC $M$, or the full AE construction $E, M$. She specifies a certain length $r$, a nonce $N$ and calls a unitary:

$$
\left|x_{1}, \ldots x_{r}\right\rangle|y, z\rangle \mapsto\left|x_{1}, \ldots x_{r}\right\rangle\left|y \oplus E_{k}\left(x_{1}, \ldots x_{r} ; N\right)\right\rangle\left|z \oplus M_{k}\left(x_{1}, \ldots x_{r} ; N\right)\right\rangle
$$

where $y$ is an $r$-block output register. The input registers $x_{1}, \ldots x_{r}$ can be in superposition, while the secret key $k$ and the nonce $N$ are both classical values. In addition, while the nonce is chosen by the adversary, it cannot be repeated. Decryption queries in superposition are considered and formally defined in [SJS16]. In that case, the decryption fails on any input in the superposition which was the result of a challenge query. This is very similar to the blinding experiment [AMRS] that we will review below. Note that a quantum attacker is free to call unkeyed primitives, such as SATURNIN-Hash or SATURNIN itself, in superposition, regardless of the security game that is considered.

IND-qCPA. For our modes of encryption, we use the notion of indistinguishability under message-superposition attacks as defined e.g. in [SJS16]. The adversary is allowed to make queries of the form (1) and classical challenge queries, in which she chooses two messages $m_{0}, m_{1}$ of equal block lengths, and the challenger replies with the encryption of one of these two messages. In order to win the game, the adversary must guess which one was encrypted. In [SJS16], the encryption mode is randomness-based and not nonce-based, but the adversary chooses the randomness, so the situation is similar to ours. 
qPRF. A quantum-secure pseudorandom function (qPRF, see e.g. [AMRS], Definition 2) is a family of functions $f_{k}: X \rightarrow Y$ indexed by a key $k$, such that any quantum polynomialtime adversary has negligible advantage in distinguishing a function $f_{k}$, with $k$ chosen at random, from a random function $f: X \rightarrow Y$. Notice that in this definition, while the adversary queries her oracle in superposition, $k$ is a classical value.

qPRP and Quantum Ideal Cipher. Similarly to a $\mathrm{qPRF}$, a cipher is a $\mathrm{qPRP}$ if no polynomial-time adversary can distinguish it, with a randomly chosen key, from a random permutation (this adversary can make encryption and decryption queries in superposition). We will also use the stronger definition of a quantum ideal cipher given in [HY18]: it is indistinguishable from a cipher chosen at random among all ciphers, under encryption and decryption queries, in superposition over the message and the key. Both definitions are extensions from the classical ideal cipher and PRP notions.

Quantum Security of MACs. To the best of our knowledge, there are two main notions of quantum unforgeability for MACs in the literature, from [BZ13a] and [AMRS] respectively. We use the notion of Blind Unforgeability (BU), which is a quantum extension of EUFCMA introduced in [AMRS]. In the blind forgery game, the adversary selects a parameter $\varepsilon$. The challenger generates a key $k$ and a random "blinding" of the MAC, $B_{\varepsilon}$, which is an $\varepsilon$-fraction of the message space on which the adversary can query the MAC, while the rest is forbidden to her. The adversary, having only access to the blinded version of the MAC, must then produce a forgery of a message $m \in B_{\varepsilon}$. This game essentially reduces to EUF-CMA when the adversary makes only classical queries.

A qPRF is a BU-secure MAC [AMRS], which is why our quantum security arguments focus on the security as a $\mathrm{qPRF}$. Notice that it also implies the SUF-qCMA security notion of [BZ13a], hence fits into the composition result of [SJS16]. The definition of the BU game in [AMRS] considers MACs without nonces, while the definition of SUF-qCMA in [SJS16] allows some randomness; since nonces are not reused, this does not make much difference.

\subsubsection{Saturnin-CTR-Cascade}

In this section, we detail the security arguments for SATURNIN-CTR-Cascade that motivate our choice of the Counter mode and Cascade.

Security of CTR. If the underlying block cipher is a PRP, the Counter mode (CTR) is classically proven secure up to the birthday bound, provided that, for any fixed key, the inputs of block cipher are never reused [Nat01]. This last condition is obviously guaranteed by choosing as input to the block cipher the concatenation of the 128-bit nonce and of a 128-bit counter. In [ATTU16], a generic proof of quantum security is provided for primitives that XOR the message to a pseudo-random sequence generated from the length of the message and a random secret key. Hence, our CTR mode being IND-CPA, it is also IND-qCPA.

Security of the Cascade Construction. We define the compression function $h(k, m)=$ $\operatorname{SATURNIN}_{k}(m) \oplus m$, where SATURNIN is used with a domain separator equal to 2, 3, 4 or 5 (but for simplicity, we do not consider the different domain separators, nor associated data). The Cascade construction builds a function $H$ from $h$ by:

$$
\left.H\left(k, m_{0}, \ldots x_{\ell}\right)=h\left(\ldots h\left(h\left(k, m_{0}\right), m_{1}\right) \ldots m_{\ell}\right)\right) .
$$

A classical proof of security for Cascade is given in [BCK96] and [Bel15]. In [SY17], it is shown that $H(k, \cdot)$ is a $\mathrm{qPRF}$ of the key $k$ if the underlying compression function $h(k, m)$ is also a qPRF. More precisely, if we fix the number $\ell$ of message blocks as a constant, 
Theorem 5.1 in [SY17] gives the advantage of a quantum adversary in distinguishing $H$ as $34 \ell q^{3 / 2} \sqrt{A}$, where $A$ is the advantage of an adversary making at most $4 q$ queries to break the PRF $h$.

Assuming that SATURNIN is a qPRP, $h(k \cdot)$ is a qPRF of $k$. Indeed, let $A$ be a distinguisher for $h(k, m)$, we can transform it into a distinguisher $B$ for $E_{k}(m)$ from a random function as follows: $B$ calls $A$. Whenever $A$ queries its oracle, we XOR $m$ to the result and return. If the oracle is a random function, feedforwarding keeps the output random.

In [Zha15], it is shown that quantumly, random functions are indistinguishable from random permutations up to the quantum birthday bound, which is $2^{n / 3}$ for $n$-bit to $n$-bit functions. The advantage of an adversary solving this problem with $q$ queries is $C \times \frac{q^{3}}{2^{n}}$ for some constant $C$.

\subsubsection{Saturnin-Short}

In [BR00], the authors prove the security (assuming a strong PRP) of an encode-thenencrypt construction. SATURNIN-Short can be seen as such, where the encoding corresponds to appending the nonce $N$, which is transmitted with the message. With small modifications, confidentiality and authenticity come from Theorems 4.1 and 4.2 in [BR00]. Quantumly, SATURNIN-Short is a qPRF of the key, which implies security against forgeries by [AMRS].

\subsubsection{Saturnin-Hash}

Contrary to SATURnin-CTR-Cascade and SATURnin-Short, the security of SATURnin-Hash does not stem from the security of SATURNIN as a PRP (resp. qPRP), but as an ideal cipher (resp. quantum ideal cipher), which is why the mode SATURNIN-Hash uses a version of SATURNIN with more rounds.

Classical Security. Classically, the security of Merkle-Damgård is related to that of the compression function. Ours uses the Matyas-Meyer-Oseas (MMO) mode, similar to the Cascade: $h_{i+1}=\operatorname{SATURNIN}_{h_{i}}\left(m_{i}\right) \oplus m_{i}$. It is dual to the Davies-Meyer construction, which injects the message block $m_{i}$ into the key, and would give rather $h_{i+1}=\operatorname{SATURNIN}_{m_{i}}\left(h_{i}\right) \oplus$ $h_{i}[\mathrm{PGV} 94]$.

Quantum Security. In [Zha18], Zhandry proves that: if the underlying compression function cannot be distinguished from a random oracle, then the Merkle-Damgård construction, provided that it uses a prefix-free encoding (hence a good padding), cannot be distinguished from a random oracle with more than negligible advantage. The compression function we use here is: $h(x, y)=\operatorname{SATURNIN}_{x}(y) \oplus y$. It is easy to see that, if SATURNIN is a quantum ideal cipher, then it is indifferentiable, as required by [Zha18]. There is also another work on the quantum security of Merkle-Damgård, instantiated with Davies-Meyer in [HY18]. We reckon that a proof such as [HY18] could be done also with the MMO mode, in the quantum ideal cipher model.

\section{Security Analysis}

\subsection{Security of the Block Cipher against Classical Attacks}

As explained in Section 5.1, the structure of the SATURNIN block cipher is very similar to the structure of an AES operating on 16-bit words. The SATURNIN block cipher then benefits from the 20-year cryptanalytic effort against the AES. Most notably, the main families of attacks against the AES can be directly transposed to SATURNIN, by replacing 
the AES 8-bit S-box by the SATURnin 16-bit Super-S-box. Due mainly to SATurnin's simplified key-schedule, we have been able to improve the highest number of attacked rounds with respect to AES, from 7 AES-rounds to 7.5 super-rounds from SATURNIN. Improved best attacks exploiting further this key-schedule might reach up to 8 super-rounds (which would already be an impressive result), but more than that seems extremely unlikely from our preliminary analysis, when considering the known cryptanalysis tools.

Differential cryptanalysis. The Super-S-box $S_{16}$ has differential uniformity 80, or equivalently the highest probability for a non-trivial differential is $80 \times 2^{-16}=2^{-9.68}$. The AES structure guarantees that any four consecutive rounds have at least 25 active SuperS-boxes. This implies that the best differential characteristics over 4 super-rounds and 8 super-rounds have probability at most $2^{-241.9}$ and $2^{-483.9}$ respectively. Moreover, it is worth noticing that the proportion of differentials for the Super-S-box with probability higher than $2^{-10}$ is very small since there are only 110 such differentials (among $2^{32}$ ); moreover, all these 110 differentials have exactly five active nibbles.

Linear cryptanalysis. The linearity of the Super-S-box is defined as the highest magnitude taken by its Walsh transform:

$$
\widehat{\mathrm{S}_{16}}(\alpha, \beta)=\sum_{x \in \mathbb{F}_{2}^{16}}(-1)^{\beta \cdot \mathrm{S}_{16}+\alpha \cdot x}, \quad \alpha, \beta \in \mathbb{F}_{2}^{16}, \beta \neq 0 .
$$

The linearity of the Super-S-box is equal to 3072, or equivalently the maximal squared correlation for a linear relation equals $3072^{2} \times 2^{-32}=2^{-8.83}$. By the same arguments as previously, we deduce that the highest squared correlation for a linear trail (aka linear potential) over 4 rounds and over 8 rounds is at most $2^{-220.7}$ and $2^{-441.5}$ respectively.

Algebraic degree. The choice of the 4-bit S-boxes $\sigma_{0}$ and $\sigma_{1}$ guarantees that all components of the Super-S-box (i.e., all non-trivial linear combinations of its coordinates) have degree 9 . The same property also holds for the inverse function, $\mathrm{S}_{16}^{-1}$, whose components have degree 9. It has been shown in [BCD11] that the degree of an SPN does not increase as fast as expected because of the structure of its S-box layer, which is composed of several transformations operating on a smaller number of variables. More precisely, when composing an S-box layer $F=(S, \ldots, S)$ with another function, an upper bound on the resulting degree can be derived from the following quantity, which characterizes the S-box:

$$
\gamma(S)=\max _{1 \leq i<m} \frac{m-i}{m-\delta_{i}}
$$

where $m$ is the size of the S-box $S$ and $\delta_{i}$ is the maximum degree of the product of $i$ coordinates of $S$. When considering the SATURNIN Super-S-box $\mathrm{S}_{16}$, this quantity $\gamma\left(\mathrm{S}_{16}\right)$ can be derived from the degree of $\mathrm{S}_{16}^{-1}$. Indeed, it is known from [BC13] that, for any $m$-bit permutation $S$, the smallest $i$ such that $\delta_{i} \geq m-1$ equals $\left(m-\operatorname{deg} S^{-1}\right)$. Here, we deduce that, for $\mathrm{S}_{16}$, we have $\delta_{7}=15$ and $\delta_{6} \leq 14$, leading to $\gamma\left(\mathrm{S}_{16}\right)=9$. We then use Theorem 2 in [BCD11] for upper-bounding the degree of two super-rounds (without the last linear layer which does not influence the degree), considered as the composition of four similar permutations $\mathrm{S}_{64}$ operating on 64 bits. Each of these permutations can be decomposed as a Super-S-box layer and a transformation of degree 9. We then deduce that $\mathrm{S}_{64}$ (and then two super-rounds) has degree at most

$$
64-\frac{64-9}{\gamma\left(\mathrm{S}_{16}\right)}=57 \text {. }
$$

Provided that this upper bound is tight both for $\mathrm{S}_{64}$ and its inverse, we can recursively apply the same arguments for proving that $\gamma\left(S_{64}\right)=57$ and that four super-rounds have 
Table 4: Upper-bound on the algebraic degree of SATURNIN when the number of superround varies.

\begin{tabular}{lccccc}
\hline$r$ (super-rounds) & 1 & 2 & 3 & 4 & 5 \\
\hline degree & 9 & 57 & 233 & 252 & 255 \\
\hline
\end{tabular}

degree at most 252. It follows that the full degree in SATURNIN is reached after at least five super-rounds. These results are summed up on Table 4.

It is worth noticing that this general upper-bound is known to provide a good estimate of the exact degree of the primitive, as shown by the experiments on Keccak [BDPA11] for instance.

Bicliques. The exhaustive search attack with bicliques [BKR11] always allows to gain a small factor against the baseline exhaustive key search, by testing all the keys faster than an evaluation of the cipher. It is applicable to SATURNIN, as to any cipher. However, with this biclique exhaustive search, it is still impossible to retrieve the correct key in less than $2^{224}$ operations, not contradicting our security claims.

Impossible Differential Attacks. Impossible differential attacks were introduced by Knudsen [Knu95] and by Biham, Biryukov and Shamir [BBS99]. They form one of the cryptanalysis families that provide some of the best known attacks on reduced-round AES [BLNS18], together with the Demirci-Selcuk MITM attacks [DS08]. They are designed by first writing an impossible differential transition, which yields a distinguisher for some middle rounds. With partial key guesses, the differential path can be extended forwards and backwards. Good guesses of the keys are such that this path cannot occur. Bad guesses make this path occur as soon as we try enough plaintext-ciphertext pairs, as the middle rounds are replaced by a random permutation. Hence, the attacker first retrieves a set of sufficiently many plaintext-ciphertext pairs with good input and output differences, and he uses these pairs to sieve the subkey space, by trying partial encryptions and decryptions, and removing the pairs that yield the impossible differential. To date, the best impossible differential attack on AES-128 [BLNS18] targets 7 rounds and it requires, with $2^{105}$ chosen plaintexts, a time of $2^{106.88}$ and memory of $2^{74}$. We estimate that a similar impossible differential attack can be applied to 7 super-rounds of SATURNIN, with twice these complexity exponents. As the key-schedule of SATURNIN is simpler than that of the AES, it may be possible to extend this attack to 7.5 super-rounds, as it is the case for DS-MITM attacks, or even 8 super-rounds, which would be a very impressive result.

Figure 21 in Appendix B presents some of the impossible differential distinguishers that we have studied. When trying to improve the number of rounds with respect to AES, we considered extending Path A two super-rounds backwards and 1.5 forwards, but we did not manage to find any configuration that did not involve the whole key, while keeping the number of needed pairs to test lower than $2^{256}$. Please note that other configurations with 3 to 1 states in the middle of the impossible path would rapidly lead to the full key being involved in the part that activates the whole state. Path B considers a part of the cipher shifted by one super-round with respect Path A. Due to the key schedule relations, this second path might be more promising to increase the number of attacked super-rounds by 0.5 or 1 , even if we have not been able so far to reach this goal.

Subspace trails. Until recently, all known distinguishers on the AES in the single-key model could reach at most 4 rounds (which would translate to 4 super-rounds of SATURNIN). However, since 2016, the first 5-round AES-distinguishers appeared [SLG+ ${ }^{+}$, GRR17, 
RBH17, Gra18]. Most notably, these distinguishers led to improved attacks on reducedround versions of the cipher, like the attack on 5 rounds described in [BDK $\left.{ }^{+} 18\right]$ based on the distinguisher exhibited in [Gra18]. The main ingredient of these distinguishers is the existence of subspace trails, i.e., of two linear subspaces $U$ and $V$ of states such that the image by the round function of any coset of $U$ is included in a coset of $V$. The previously mentioned results on the AES exploit such subspace trails over two rounds of the cipher. The length of the longest subspace trail for a cipher is therefore an essential quantity in these attacks. However, it has been shown in [LTW18] that, if the S-box does not have any linear structure (i.e. any component with a constant differential), then all subspace trails are the direct product of subspace trails of the single S-box, i.e. in our case, the direct product of 16 subspaces, each of them being either $\{0\}$ or $\mathbb{F}_{2}^{16}$. It can easily be checked that the Super-S-box $S_{16}$ does not have any linear structure, implying that the previous property is valid. Then, SATURNIN behaves exactly as the AES with respect to subspace trails: the fact that the linear layer in the Super-S-box representation consists of the multiplication of each column in Matrix $C$ by an MDS matrix over $\mathbb{F}_{2}^{16}$ implies that the longest subspace trail has a length corresponding to two super-rounds and is obtained by considering as input linear space a collection of columns of the matrix (i.e., a collection of slices in the cube representation). It follows that any distinguisher or attack exploiting this property cannot reach more than five super-rounds.

\subsection{DS-MITM attack on 7.5-super-round Saturnin}

The DS-MITM attack [DS08, DFJ13] yields the best reduced-round single-key recoveries on AES to date. There exists a variant for each key length of AES. Indeed, the attack works by guessing parts of the internal state; hence the ratio between the state size and the key size is important, and also, the key-schedule relations in AES depend on the key size. In our case, the key size is equal to the block size, so SATURNIN is more analogous to AES-128 than the others. The best DS-MITM attack on AES-128 reaches 7 rounds, runs in data $2^{105}$, time $2^{105}$ and memory $2^{81}$. It works using a distinguisher on the middle rounds with the following property: given a constrained input and output differential, the values of the internal states can only take few possibilities; if we make the input vary, the sequence of outputs stays in a limited subspace. It is possible to tabulate all these possibilities (in the simple AES-128 attack, there are $2^{80}$ of them). The adversary finds pairs with some input-output difference. She guesses part of the key, allowing a pair to satisfy the whole inner differential path, and checks whether the middle property is satisfied for this pair. This fails for all subkey guesses, except the right one, since the middle property is very constrained. The 7-round attack works against SATURNIN, with twice the complexity exponents.

We now present an improved DS-MITM on $\mathbf{7 . 5}$ super-rounds, where the improvement compared to AES comes from the simpler key-schedule. It is, as could be expected, the best known cryptanalysis on a reduced-round version of SATURNIN. We rewrite SATURNIN as a $4 \times 4$ square of 16 -bit "supernibbles" which correspond to columns in the cube. We write the Super-S-box as $S$, which is actually an S-box, followed by MixColumns, followed by another S-box. In even rounds, the key is XORed to the internal state; in odd rounds, it is rotated by 5 supernibble-positions (they correspond to the 20 nibble-positions by which the key is rotated in the cube representation of SATURNIN).

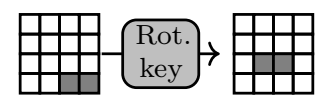

In the differential path that we use for 7.5-super-round SATURNIN, the plaintexts are active in a single row, while the ciphertexts are active in three lines. We will in total guess 15 supernibbles of the key, corresponding to the intersection of three lines and three 


\begin{tabular}{|c|c|c|c|}
\hline 0 & 4 & 8 & 12 \\
\hline 1 & 5 & 9 & 13 \\
\hline 2 & 6 & 10 & 14 \\
\hline 3 & 7 & 11 & 15 \\
\hline
\end{tabular}

Figure 18: AES byte ordering

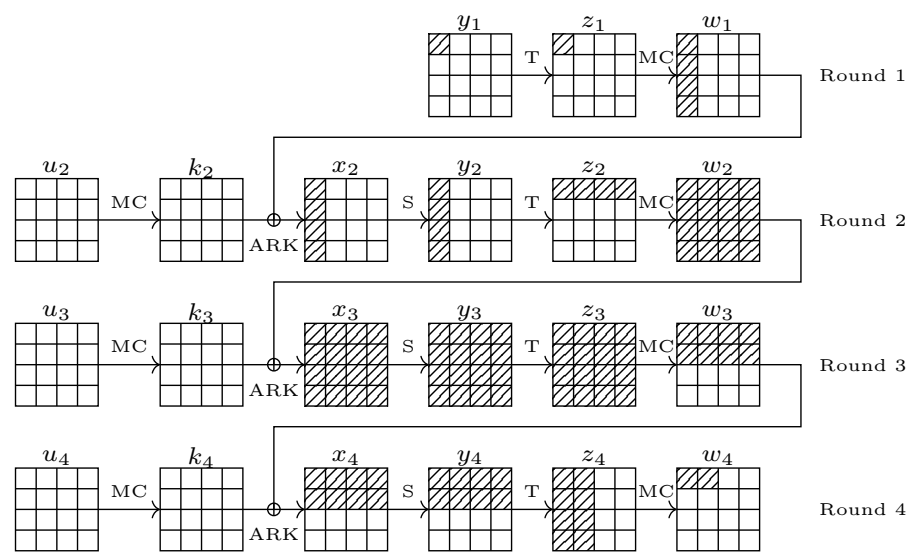

Figure 19: Differential path used in the "internal" property of our DS-MITM attack on 7.5-super-round SATURNIN.

columns. In the following, for more clarity, we number the supernibbles with the standard AES byte numbering. The sequence of states of round $i$ is denoted $x_{i}$ (after adding the key), $y_{i}$ (after the Super-S-box), $z_{i}$ (after transposition) and $w_{i}$ (after Super-Mixcolumns).

The attack uses the following property (see Figure 19), which is a variant of the usual middle-rounds property for the DS-MITM attacks on AES: if we are given a plaintextciphertext pair such that in $y_{1}$, only the nibble 0 is active and, in $w_{4}$, only the nibbles 0,4 are active, then if we make $y_{1}[0]$ take a sequence of $2^{4}$ arbitrary differences and obtain the corresponding 32 -bit differences in $w_{4}[0,4]$, they can only take up to $2^{16 \times(4+8+2+1)}=2^{240}$ values among $2^{32 \times 2^{4}}$. This allows to efficiently distinguish between 4 AES rounds and a random cipher.

The proof of this property uses arguments inherited from rebound attacks [MRST09]. We first guess the value of $\Delta y_{1}[0]$, the difference in $y_{1}[0]$, for the given pair. We obtain the difference in $x_{2}$. We guess the four nibbles $x_{2}[0,1,2,3]$ and obtain the differences in $y_{2}$, hence $x_{3}$. On the other side, we guess $\Delta w_{4}[0,4]$, hence obtaining $\Delta y_{4}$; we guess 8 more state nibbles to obtain $\Delta x_{4}$, hence $\Delta y_{3}$. It remains to match between the two differences. There is on average one solution (we assume that the solutions to the differential equation of the Super-S-box are tabulated). At this point, we know the whole sequence of states $x_{2}[0,1,2,3], x_{3}[0 \ldots 15], x_{4}[0,1,4,5,8,9,12,13]$ for the given pair. So we can propagate the arbitrary sequence of differences in $y_{1}[0]$ up to $w_{4}[0,4]$, whose values can be computed and stored.

Attack. We use the usual DS-MITM attack principle, but reorder the steps as done in the last section of [BNPS19]. First of all, using $2^{225}$ encryption queries (grouped into structures that make the first line of the input take all $2^{64}$ possibilities), we build $2^{14 \times 16}=2^{224}$ plaintext-ciphertext pairs verifying the input-output differential. We then build a table of size $2^{15 \times 16}=2^{240}$, indexed by all $2^{240}$ guesses of $K$ (the whole key, except the nibble number 3 ) and associated to good pairs of plaintext. The rest of the computation consists 


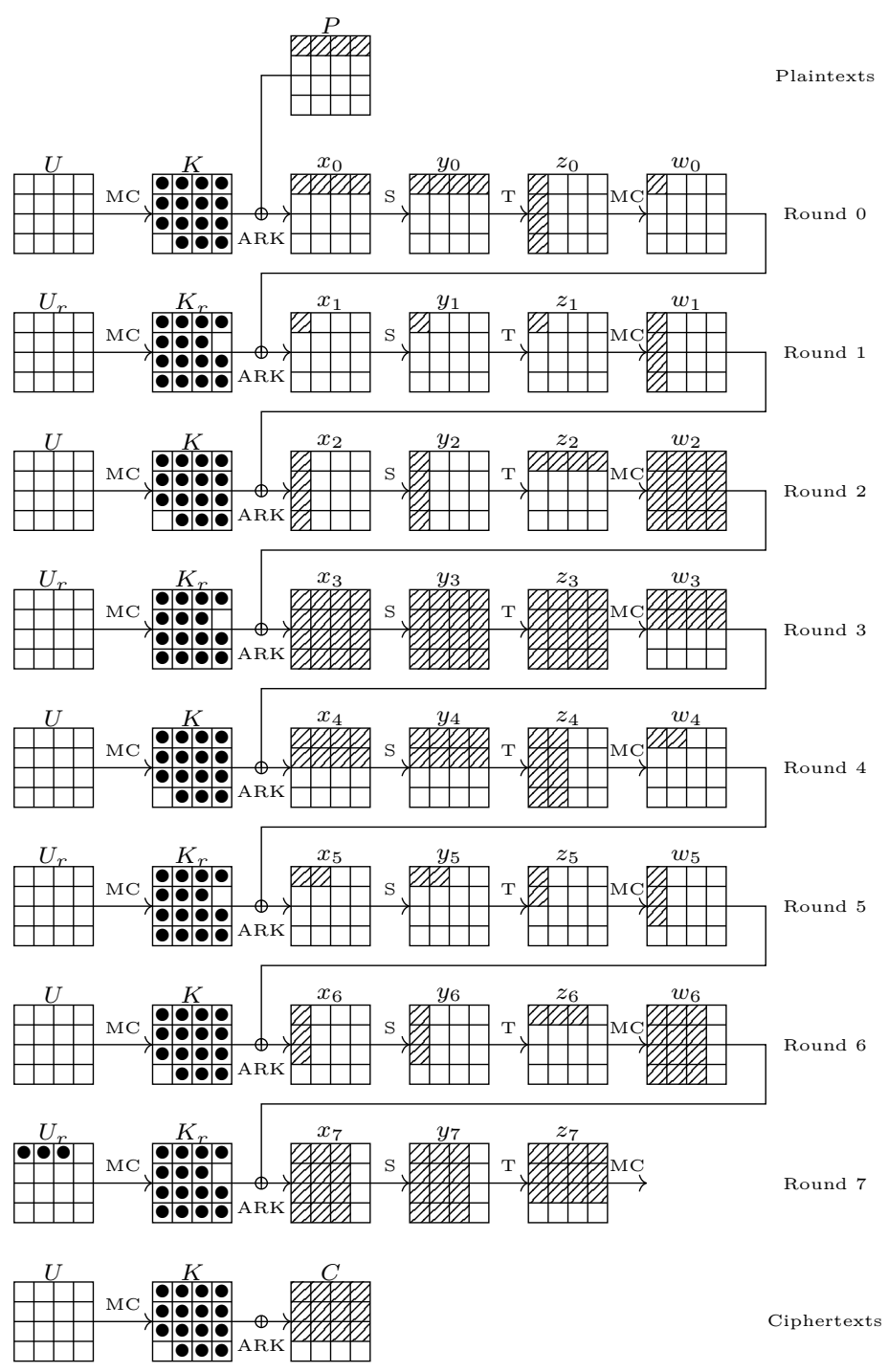

Figure 20: Full differential path used in the DS-MITM attack on 7.5-super-round SatURNIN. We denote the master key $K$ and its rotated version (for odd rounds) $K_{r} . U$ and $U_{r}$ are respectively $M C^{-1}(K)$ and $M C^{-1}\left(K_{r}\right)$. 
in finding the good key guess in this table:

- For each of the $2^{224}$ pairs, we find the $2^{16}$ key guesses such that the whole differential path is satisfied: we have 3 supernibbles conditions in round 0 in order to reach the input of the internal property, while we have $(9+2)$ supernibble conditions in rounds 6 and 5 respectively for reaching the output of the internal property. As we have 15 supernibbles of the key involved and a total of 14 supernibble conditions, we obtain around $2^{16}$ posibilities for the 15 words of the key that lead to the internal property. In less than $2^{240}$ time, thanks to early-abort techniques [LKKD08], we associate each key guess, with the good corresponding pairs leading to the correct path.

- Consider a key guess and its corresponding pair. Let $P, x_{0} \ldots z_{7}, C$ and $P^{\prime}, x_{0}^{\prime} \ldots z_{7}^{\prime}, C^{\prime}$ be the respective sequences of internal states for this pair. We create a set of $2^{4}$ plaintexts by making the difference in $y_{1}[0]$ assume the $2^{4}$ arbitrary values chosen before. Due to the knowledge of the key supernibbles, we know the value of $y_{1}[0]$, so we can propagate its difference to $w_{0}[0]$, and know the necessary state nibbles to complete our plaintext sequence. We encrypt this sequence with the secret-key oracle, obtaining $2^{4}$ ciphertexts. We then partially decrypt the corresponding ciphertexts and obtain the sequence of differences in $w_{4}[0,4]$, since we know the necessary key supernibbles. In total, this requires $2^{244}$ encryptions, after which we have stored $2^{244}$ 256-bit blocks with the associated keys.

- Then, we perform an exhaustive search in the middle. We compute all possible sequences of $2^{4}$ differences in $w_{4}[0,4]$, for all choices of internal state supernibbles, and we search a collision with some key guess in the table. There are $2^{240}$ sequences to compute, each costs $2^{4}$ partial SATURNin encryptions.

In the end, we expect only one collision to occur, which gives the good key guess (that we can complete easily after). This slightly improved attack mainly works because of the simpler key-schedule of SATURNIN, which allows to reuse multiple times the key guesses, contrary to AES-128. The attack complexities are $2^{244}$ chosen-plaintext queries, an equivalent time, and $2^{244}$ memory (counted in 256-bit blocks).

\subsection{Security of the Block Cipher Against Quantum Attacks}

Given a few plaintext-ciphertext pairs, a quantum adversary can perform a naive exhaustive search for the key using Grover's algorithm [Gro96]. It requires approximately $2^{256 / 2}=2^{128}$ iterations (we claim that it requires more than $2^{112}$ encryptions), each of which contains basic operations and an evaluation of an implementation of SATURNIN as a quantum circuit (see [GLRS16] for the AES). We do not go into the details of such an implementation, but we point out that it would require at least as many quantum gates as classical gates (and possibly more, due to the necessity of reversibility), with interleaved layers of quantum error correction. Error-correcting operations are quantum, but classically controlled, and they provoke an overhead with respect to classical computations.

In its super-S-box representation, SATURNIN has shape similar to AES, with a 256-bit state. To date, there does not exist much literature on quantum attacks on reduced-round AES in the secret-key setting, i.e. procedures that retrieve the secret key faster than Grover's algorithm. A first analysis was made in [BNPS19]. The authors show that quantum versions of the Square attack can target up to 6 rounds of AES-128 and 7 rounds of AES-192 and AES-256 (as it is the case classically), and they construct a quantum DS-MITM attack on 8-round AES-256. All these procedures only require classical plaintext queries to a secret-key oracle. No attack was found with a better speed-up than quadratic, so for now it seems safe to claim, at least, the same quantum security margin as the classical one. 
We should remark here that the DS-MITM attack of [BNPS19] reaches 8 rounds in a case where the key length is the double of the block length. On the contrary, the quantum security margin of SATURNIN, the highest number of rounds attacked with a procedure faster than Grover, would likely be similar to that of AES-128. Indeed, we do not know of any better quantum attack than an adaptation of the Square for 6-round AES (its time, data and memory complexity being roughly the square of the original ones, given in Table 3 of [BNPS19]). The quantum security margin seems therefore bigger, with 6 reached super-rounds versus 7.5 classically.

We also remark that computing input-output pairs with target differences is the bottleneck of classical impossible differential and DS-MITM attacks against AES-128. This operation seems to enjoy less than a quadratic quantum speedup, as this requires finding partial collisions [BHT98, Amb07], and in some cases, it may require high amounts of quantum memory. Hence it may be difficult to make quantum versions of these attacks competitive against an exhaustive search for the key. The attack of [BNPS19] actually overcomes this issue by using only a low amount of classically computed pairs.

\subsection{Security of the Modes of Operation}

In this section, we summarize the best attacks known against SATURNIN-CTR-Cascade, SATURNin-Short and SATURnin-Hash. As these attacks are generic, we first detail some generic variants of quantum exhaustive search. We do not consider parallelization (classical nor quantum).

Quantum Search with Limited Time. Let $f:\{0,1\}^{n} \rightarrow\{0,1\}$ be a random oracle, such that 1 has a single preimage, and let us look for this preimage with superposition access to $f$, with a time limitation $\mathcal{T}$. By running Grover's algorithm for $\mathcal{T}$ steps, we obtain the good result with success probability $\frac{\mathcal{T}^{2}}{2^{n}}$, instead of $\frac{\mathcal{T}}{2^{n}}$ classically. Both are optimal. As each iteration uses a single query to $f$, if we put a data limitation $\mathcal{D}$ instead, we obtain a probability $\frac{\mathcal{D}^{2}}{2^{n}}$. This is also why quantum exhaustive search has a worse parallelization speedup than its classical counterpart: stopping Grover's algorithm after some time makes the success probability decrease quadratically.

Quantum Collision Search with Limited Memory. Let $f:\{0,1\}^{n} \rightarrow\{0,1\}^{n}$ be a random oracle, with superposition query access. An algorithm by Brassard, Høyer and Tapp [BHT98] allows to retrieve a collision of $f$ in approximately $2^{n / 3}$ superposition queries, using $2^{n / 3}$ classical memory with quantum random access. But this model seems currently far away from practical realizations. Some authors [GR04, Ber09, JS19] argue that a quantity $\mathcal{M}_{q}$ of such memory would certainly require $O\left(\mathcal{M}_{q}\right)$ classical computations at each step, due to quantum error correction, reducing significantly its practical advantages. Without quantum random-access, an alternative collision search can be done using [CNS17] in $2^{2 n / 5}$ superposition queries, using only as many qubits as required by Grover iterations and oracle evaluations. It is worth noticing that, while the query lower bound for random functions is known to be $2^{n / 3}$, there exists no proof that better memory usage than [BHT98] or better time complexity than [CNS17] is impossible, but no such algorithm is known. We subsume [CNS17] and [BHT98] with a single (maybe non tight) bound of $\mathcal{T}^{5} \times \mathcal{M}_{q}=2^{2 n}$ where $\mathcal{M}_{q}$ is the quantum memory available and $\mathcal{T}$ is the quantum time complexity.

Quantum Collision Search with Limited Data. With $\mathcal{D}$ quantum queries, quantum collision search succeeds with an optimal probability $\frac{\mathcal{D}^{3}}{2^{n}}$ [Zha15]. The best method consists in applying a truncated version of [BHT98]: we first query $\mathcal{D} / 2$ elements, store them in quantum-accessible memory and run $\mathcal{D} / 2$ iterates of Grover's algorithm, searching for a match on this intermediate table. 
Classical Attacks against Saturnin-CTR-Cascade. Let $t$ be the tag length and $p$ the probability of success of a classical adversary against SATURNIN-CTR-Cascade in breaking its confidentiality, integrity or authenticity. We have:

$$
p<\frac{\mathcal{T}}{2^{256}}+\frac{\mathcal{D}^{2}}{2^{256}}+\mathcal{D} 2^{-t}
$$

where the adversary is entitled to encryption or verification queries of a total of $\mathcal{D}$ blocks, and $\mathcal{T}$ computation time. Indeed, the adversary succeeds in breaking the PRP security of SATURnin, i.e. in finding the key, with probability $\frac{\mathcal{T}}{2^{256}}$ if she has time $\mathcal{T}$. She can output a collision after $\mathcal{D}$ encryption queries with probability $\frac{\mathcal{D}^{2}}{2^{256}}$, breaking the AE scheme as a PRF. Finally, she can simply ask verification queries of random ciphertexts and tags. Each of them has a probability $2^{-t}$ of being accepted: the verifier will decipher and recompute the corresponding tag, ending up with a random string that must match the adversary's tag. Hence the probability of success after $\mathcal{D}$ verification queries is $\mathcal{D} 2^{-t}$.

Quantum Attacks against Saturnin-CTR-Cascade. With the same notations as above, we obtain:

$$
p<\frac{\mathcal{T}^{2}}{2^{256}}+\frac{\mathcal{D}^{3}}{2^{256}}+\mathcal{D}^{2} 2^{-t}
$$

where the adversary is entitled to encryption or verification queries of a total of $\mathcal{D}$ blocks (in superposition), and $\mathcal{T}$ computation time. Indeed, the adversary succeeds in breaking the PRP security of the block cipher, with probability $\frac{\mathcal{T}^{2}}{2^{n}}$ if she has time $\mathcal{T}$ (see Section 5.3). Furthermore, she succeeds in breaking the AE scheme as a PRF with probability $\frac{\mathcal{D}^{3}}{2^{n}}$, since this is the advantage of outputting a collision after $\mathcal{D}$ queries. Finally, she succeeds with probability $\mathcal{D}^{2} 2^{-t}$ in making the verifier accept a random tag, using Grover's algorithm with the verifier as oracle.

Attacks against Saturnin-Short. In SATURNIN-Short, the generic attacks are slightly different from SATURNIN-CTR-Cascade. Although the tag is not truncated to 128 bits, the attack using verification queries applies as if it were the case. Indeed, a forgery using verification queries amounts to finding $c, N$ such that $E_{k}^{-1}(c)$ contains $N$ on its 128 right bits. $N$ is not queried in superposition, and the adversary has only access to the verification result, not the value. So this amounts to looking for $2^{256}$ good elements (sound pairs $c, N$ ) among $2^{256+128}$ (all choices) with the verifier as oracle. Classically, the probability of success after $\mathcal{D}$ trials is $\mathcal{D} 2^{-128}$.

Quantumly, Grover's algorithm speeds up the search for a random ciphertext and nonce giving a good verification result. With $\mathcal{D}$ superposition verification queries, the success probability is $\mathcal{D}^{2} 2^{-128}$, so everything happens as if the tag was actually truncated to 128 bits.

Attacks against Saturnin-Hash. The best known attacks (finding a collision or a preimage) are the generic attacks given in Section 5.3. The best time is $2^{85}$ with a quantumaccessible memory of size $2^{85}$. Without quantum-accessible memory, the best quantum time known is $2^{102}$, given by [CNS17].

\section{Acknowledgements}

The authors would like to thank the reviewers, especially Joan Daemen for his careful reading of this paper. They are also particularly grateful to Xavier Bonnetain for very helpful discussions and comments, and in particular for an invaluable remark detecting a weakness on a preliminary version of SATURNIN. This project has received funding from 
the European Research Council (ERC) under the European Union's Horizon 2020 research and innovation programme (grant agreement no. 714294 - acronym QUASYModo).

\section{References}

$\left[\mathrm{ADK}^{+} 14\right]$ Martin R. Albrecht, Benedikt Driessen, Elif Bilge Kavun, Gregor Leander, Christof Paar, and Tolga Yalçin. Block ciphers - focus on the linear layer (feat. PRIDE). In Juan A. Garay and Rosario Gennaro, editors, CRYPTO 2014 , Part I, volume 8616 of $L N C S$, pages 57-76. Springer, Heidelberg, August 2014.

[Amb07] Andris Ambainis. Quantum walk algorithm for element distinctness. SIAM J. Comput., 37(1):210-239, 2007.

[AMRS] Gorjan Alagic, Christian Majenz, Alexander Russell, and Fang Song. Quantum-secure message authentication via blind-unforgeability. IACR Cryptology ePrint Archive, Report 2018/1150. https://eprint.iacr.org/2018/ 1150 .

[ATTU16] Mayuresh Vivekanand Anand, Ehsan Ebrahimi Targhi, Gelo Noel Tabia, and Dominique Unruh. Post-quantum security of the CBC, CFB, OFB, CTR, and XTS modes of operation. In Tsuyoshi Takagi, editor, Post-Quantum Cryptography - 7th International Workshop, PQCrypto 2016, pages 44-63. Springer, Heidelberg, 2016.

[BAK98] Eli Biham, Ross J. Anderson, and Lars R. Knudsen. Serpent: A new block cipher proposal. In Serge Vaudenay, editor, FSE'98, volume 1372 of LNCS, pages 222-238. Springer, Heidelberg, March 1998.

[BBS99] Eli Biham, Alex Biryukov, and Adi Shamir. Cryptanalysis of Skipjack reduced to 31 rounds using impossible differentials. In Jacques Stern, editor, EUROCRYPT'99, volume 1592 of LNCS, pages 12-23. Springer, Heidelberg, May 1999.

[BC13] Christina Boura and Anne Canteaut. On the influence of the algebraic degree of $F^{-1}$ on the algebraic degree of $G \circ F$. IEEE Trans. Information Theory, 59(1):691-702, 2013.

[BCD11] Christina Boura, Anne Canteaut, and Christophe De Cannière. Higher-order differential properties of Keccak and Luffa. In Antoine Joux, editor, FSE 2011, volume 6733 of LNCS, pages 252-269. Springer, Heidelberg, February 2011.

[BCK96] Mihir Bellare, Ran Canetti, and Hugo Krawczyk. Keying hash functions for message authentication. In Neal Koblitz, editor, CRYPTO'96, volume 1109 of $L N C S$, pages 1-15. Springer, Heidelberg, August 1996.

[BDK $\left.{ }^{+} 18\right]$ Achiya Bar-On, Orr Dunkelman, Nathan Keller, Eyal Ronen, and Adi Shamir. Improved key recovery attacks on reduced-round AES with practical data and memory complexities. In Hovav Shacham and Alexandra Boldyreva, editors, CRYPTO 2018, Part II, volume 10992 of LNCS, pages 185-212. Springer, Heidelberg, August 2018.

$\left[\mathrm{BDP}^{+} 12\right]$ Guido Bertoni, Joan Daemen, Mickaël Peeters, Gilles Van Assche, and Ronny Van Keer. The Keccak implementation overview. https://keccak. team/files/Keccak-implementation-3.2.pdf, 2012. 
[BDPA11] Guido Bertoni, Joan Daemen, Mickaël Peeters, and Gilles Van Assche. The Keccak reference. Submission to the SHA-3 competition (round 3), 2011. https://keccak.team/files/Keccak-reference-3.0.pdf.

[Bel15] Mihir Bellare. New proofs for NMAC and HMAC: Security without collision resistance. Journal of Cryptology, 28(4):844-878, October 2015.

[Ber09] Daniel J. Bernstein. Cost analysis of hash collisions: Will quantum computers make SHARCS obsolete. In SHARCS 2009, pages 105-116, 2009. Available at http://skew2011.mat.dtu.dk/proceedings/.

[BHT98] Gilles Brassard, Peter Høyer, and Alain Tapp. Quantum cryptanalysis of hash and claw-free functions. In Claudio L. Lucchesi and Arnaldo V. Moura, editors, LATIN 1998, volume 1380 of LNCS, pages 163-169. Springer, Heidelberg, April 1998.

[Bih97] Eli Biham. A fast new DES implementation in software. In Eli Biham, editor, FSE'97, volume 1267 of LNCS, pages 260-272. Springer, Heidelberg, January 1997.

[BJK $\left.{ }^{+} 16\right]$ Christof Beierle, Jérémy Jean, Stefan Kölbl, Gregor Leander, Amir Moradi, Thomas Peyrin, Yu Sasaki, Pascal Sasdrich, and Siang Meng Sim. The SKINNY family of block ciphers and its low-latency variant MANTIS. In Matthew Robshaw and Jonathan Katz, editors, CRYPTO 2016, Part II, volume 9815 of $L N C S$, pages 123-153. Springer, Heidelberg, August 2016.

[BK03] Mihir Bellare and Tadayoshi Kohno. A theoretical treatment of related-key attacks: RKA-PRPs, RKA-PRFs, and applications. In Eli Biham, editor, EUROCRYPT 2003, volume 2656 of LNCS, pages 491-506. Springer, Heidelberg, May 2003.

[BK09] Alex Biryukov and Dmitry Khovratovich. Related-key cryptanalysis of the full AES-192 and AES-256. In Mitsuru Matsui, editor, ASIACRYPT 2009, volume 5912 of $L N C S$, pages 1-18. Springer, Heidelberg, December 2009.

[BKN09] Alex Biryukov, Dmitry Khovratovich, and Ivica Nikolic. Distinguisher and related-key attack on the full AES-256. In Shai Halevi, editor, CRYPTO 2009, volume 5677 of LNCS, pages 231-249. Springer, Heidelberg, August 2009.

[BKR11] Andrey Bogdanov, Dmitry Khovratovich, and Christian Rechberger. Biclique cryptanalysis of the full AES. In Dong Hoon Lee and Xiaoyun Wang, editors, ASIACRYPT 2011, volume 7073 of LNCS, pages 344-371. Springer, Heidelberg, December 2011.

[BLNS18] Christina Boura, Virginie Lallemand, María Naya-Plasencia, and Valentin Suder. Making the impossible possible. Journal of Cryptology, 31(1):101-133, January 2018.

[BN00] Mihir Bellare and Chanathip Namprempre. Authenticated encryption: Relations among notions and analysis of the generic composition paradigm. In Tatsuaki Okamoto, editor, ASIACRYPT 2000, volume 1976 of LNCS, pages 531-545. Springer, Heidelberg, December 2000.

[BN08] Mihir Bellare and Chanathip Namprempre. Authenticated encryption: Relations among notions and analysis of the generic composition paradigm. Journal of Cryptology, 21(4):469-491, October 2008. 
[BNPS19] Xavier Bonnetain, María Naya-Plasencia, and André Schrottenloher. Quantum security analysis of AES. IACR Cryptology ePrint Archive, Report 2019/272, 2019. https://eprint.iacr.org/2019/272.

[BR00] Mihir Bellare and Phillip Rogaway. Encode-then-encipher encryption: How to exploit nonces or redundancy in plaintexts for efficient cryptography. In Tatsuaki Okamoto, editor, ASIACRYPT 2000, volume 1976 of LNCS, pages 317-330. Springer, Heidelberg, December 2000.

[BSS ${ }^{+}$13] Ray Beaulieu, Douglas Shors, Jason Smith, Stefan Treatman-Clark, Bryan Weeks, and Louis Wingers. The SIMON and SPECK families of lightweight block ciphers. Cryptology ePrint Archive, Report 2013/404, 2013. http: //eprint.iacr.org/2013/404.

[BZ13a] Dan Boneh and Mark Zhandry. Quantum-secure message authentication codes. In Thomas Johansson and Phong Q. Nguyen, editors, EUROCRYPT 2013, volume 7881 of $L N C S$, pages 592-608. Springer, Heidelberg, May 2013.

[BZ13b] Dan Boneh and Mark Zhandry. Secure signatures and chosen ciphertext security in a quantum computing world. In Ran Canetti and Juan A. Garay, editors, CRYPTO 2013, Part II, volume 8043 of LNCS, pages 361-379. Springer, Heidelberg, August 2013.

$\left[\mathrm{CHP}^{+} 17\right]$ Carlos Cid, Tao Huang, Thomas Peyrin, Yu Sasaki, and Ling Song. A security analysis of Deoxys and its internal tweakable block ciphers. IACR Trans. Symm. Cryptol., 2017(3):73-107, 2017.

[CNS17] André Chailloux, María Naya-Plasencia, and André Schrottenloher. An efficient quantum collision search algorithm and implications on symmetric cryptography. In Tsuyoshi Takagi and Thomas Peyrin, editors, ASIACRYPT 2017, Part II, volume 10625 of LNCS, pages 211-240. Springer, Heidelberg, December 2017.

[Dae95] Joan Daemen. Cipher and hash function design, strategies based on linear and differential cryptanalysis. PhD thesis, K.U. Leuven, 1995. http://jda. noekeon.org/.

[Dam90] Ivan Damgård. A design principle for hash functions. In Gilles Brassard, editor, CRYPTO'89, volume 435 of $L N C S$, pages 416-427. Springer, Heidelberg, August 1990.

[DFJ13] Patrick Derbez, Pierre-Alain Fouque, and Jérémy Jean. Improved key recovery attacks on reduced-round AES in the single-key setting. In Thomas Johansson and Phong Q. Nguyen, editors, EUROCRYPT 2013, volume 7881 of LNCS, pages 371-387. Springer, Heidelberg, May 2013.

[DGV94] Joan Daemen, René Govaerts, and Joos Vandewalle. A new approach to block cipher design. In Ross J. Anderson, editor, FSE'93, volume 809 of LNCS, pages 18-32. Springer, Heidelberg, December 1994.

[DKR97] Joan Daemen, Lars R. Knudsen, and Vincent Rijmen. The block cipher Square. In Eli Biham, editor, FSE'97, volume 1267 of LNCS, pages 149-165. Springer, Heidelberg, January 1997.

[DL18] Sébastien Duval and Gaëtan Leurent. MDS matrices with lightweight circuits. IACR Trans. Symm. Cryptol., 2018(2):48-78, 2018. 
[DPVAR00] Joan Daemen, Michaël Peeters, Gilles Van Assche, and Vincent Rijmen. Nessie proposal: NOEKEON. First Open NESSIE Workshop, see also http: //gva.noekeon.org/papers/2000-NESSIE-Noekeon-Spec.pdf, 2000.

[DR99] Joan Daemen and Vincent Rijmen. AES Proposal: Rijndael. Submission to the NIST AES competition, 1999.

[DR02a] Joan Daemen and Vincent Rijmen. AES and the wide trail design strategy (invited talk). In Lars R. Knudsen, editor, EUROCRYPT 2002, volume 2332 of LNCS, pages 108-109. Springer, Heidelberg, April / May 2002.

[DR02b] Joan Daemen and Vincent Rijmen. The Design of Rijndael: AES - The Advanced Encryption Standard. Information Security and Cryptography. Springer, 2002.

[DS08] Hüseyin Demirci and Ali Aydin Selçuk. A meet-in-the-middle attack on 8-round AES. In Kaisa Nyberg, editor, FSE 2008, volume 5086 of LNCS, pages 116-126. Springer, Heidelberg, February 2008.

[Gag17] Tommaso Gagliardoni. Quantum Security of Cryptographic Primitives. PhD thesis, Darmstadt University of Technology, Germany, 2017.

$\left[\mathrm{GBB}^{+} 08\right]$ Henri Gilbert, Ryad Benadjila, Olivier Billet, Gilles Macario-Rat, Thomas Peyrin, Matt Robshaw, and Yannick Seurin. SHA-3 proposal: ECHO. Submission to NIST, 2008. https://ehash.iaik.tugraz.at/uploads/9/91/ Echo.pdf.

[GHS16] Tommaso Gagliardoni, Andreas Hülsing, and Christian Schaffner. Semantic security and indistinguishability in the quantum world. In Matthew Robshaw and Jonathan Katz, editors, CRYPTO 2016, Part III, volume 9816 of LNCS, pages 60-89. Springer, Heidelberg, August 2016.

[GJ75] Terry Gilliam and Terry Jones. Monty Python and the Holy Grail. Distributed by EMI Films, 1975.

$\left[\mathrm{GKM}^{+} 08\right]$ Praveen Gauravaram, Lars R. Knudsen, Krystian Matusiewicz, Florian Mendel, Christian Rechberger, Martin Schläffer, and Søren S. Thomsen. Grøstl - a SHA-3 candidate. Submission to NIST, 2008. http://www. groestl. info/ Groestl.pdf.

[GLRS16] Markus Grassl, Brandon Langenberg, Martin Roetteler, and Rainer Steinwandt. Applying Grover's algorithm to AES: Quantum resource estimates. In Tsuyoshi Takagi, editor, Post-Quantum Cryptography - 7th International Workshop, PQCrypto 2016, pages 29-43. Springer, Heidelberg, 2016.

[GM08] Samuel Galice and Marine Minier. Improving integral attacks against Rijndael256 up to 9 rounds. In Serge Vaudenay, editor, AFRICACRYPT 08, volume 5023 of LNCS, pages 1-15. Springer, Heidelberg, June 2008.

[GR04] Lov K. Grover and Terry Rudolph. How significant are the known collision and element distinctness quantum algorithms? Quantum Information 83 Computation, 4(3):201-206, 2004.

[Gra18] Lorenzo Grassi. Mixture differential cryptanalysis: a new approach to distinguishers and attacks on round-reduced AES. IACR Trans. Symm. Cryptol., 2018(2):133-160, 2018. 
[Gro96] Lov K. Grover. A fast quantum mechanical algorithm for database search. In 28th ACM STOC, pages 212-219. ACM Press, May 1996.

[GRR17] Lorenzo Grassi, Christian Rechberger, and Sondre Rønjom. A new structuraldifferential property of 5-round AES. In Jean-Sébastien Coron and Jesper Buus Nielsen, editors, EUROCRYPT 2017, Part II, volume 10211 of LNCS, pages 289-317. Springer, Heidelberg, April / May 2017.

[HY18] Akinori Hosoyamada and Kan Yasuda. Building quantum-one-way functions from block ciphers: Davies-Meyer and Merkle-Damgård constructions. In Thomas Peyrin and Steven Galbraith, editors, ASIACRYPT 2018, Part I, volume 11272 of $L N C S$, pages 275-304. Springer, Heidelberg, December 2018.

$\left[\mathrm{IAC}^{+}\right.$08] Sebastiaan Indesteege, Elena Andreeva, Christophe De Cannière, Orr Dunkelman, Emilia Käsper, Svetla Nikova, Bart Preneel, and Elmar Tischhauser. The LANE hash function. Submission to NIST, 2008. https://www.esat . kuleuven.be/cosic/publications/article-1181.pdf.

[JS19] Samuel Jaques and John M. Schanck. Quantum cryptanalysis in the RAM model: Claw-finding attacks on SIKE. IACR Cryptology ePrint Archive, Report 2019/103, 2019. https://eprint.iacr.org/2019/103.

[KLLN16a] Marc Kaplan, Gaëtan Leurent, Anthony Leverrier, and María Naya-Plasencia. Breaking symmetric cryptosystems using quantum period finding. In Matthew Robshaw and Jonathan Katz, editors, CRYPTO 2016, Part II, volume 9815 of $L N C S$, pages 207-237. Springer, Heidelberg, August 2016.

[KLLN16b] Marc Kaplan, Gaëtan Leurent, Anthony Leverrier, and María Naya-Plasencia. Quantum differential and linear cryptanalysis. IACR Trans. Symm. Cryptol., 2016(1):71-94, 2016. http://tosc.iacr.org/index.php/ToSC/article/ view/536.

[KM10] Hidenori Kuwakado and Masakatu Morii. Quantum distinguisher between the 3-round Feistel cipher and the random permutation. In ISIT 2010, pages 2682-2685. IEEE, 2010.

[KM12] Hidenori Kuwakado and Masakatu Morii. Security on the quantum-type even-mansour cipher. In ISITA 2012, pages 312-316. IEEE, 2012.

[Knu95] Lars R. Knudsen. Truncated and higher order differentials. In Bart Preneel, editor, FSE'94, volume 1008 of LNCS, pages 196-211. Springer, Heidelberg, December 1995.

[LKKD08] Jiqiang Lu, Jongsung Kim, Nathan Keller, and Orr Dunkelman. Improving the efficiency of impossible differential cryptanalysis of reduced Camellia and MISTY1. In Tal Malkin, editor, CT-RSA 2008, volume 4964 of $L N C S$, pages 370-386. Springer, Heidelberg, April 2008.

[LP07] Gregor Leander and Axel Poschmann. On the Classification of 4 Bit S-Boxes. In Claude Carlet and Berk Sunar, editors, WAIFI 200\%, volume 4547 of LNCS, pages 159-176. Springer, Heidelberg, June 2007.

[LTW18] Gregor Leander, Cihangir Tezcan, and Friedrich Wiemer. Searching for subspace trails and truncated differentials. IACR Trans. Symm. Cryptol., 2018(1):74-100, 2018. 
[Mer90] Ralph C. Merkle. A certified digital signature. In Gilles Brassard, editor, CRYPTO'89, volume 435 of LNCS, pages 218-238. Springer, Heidelberg, August 1990.

[MMO85] Stephen M. Matyas, Carl H. Meyer, and Jeffrey M. Oseas. Generating strong one-way functions with cryptographic algorithm. IBM Technical Disclosure Bulletin 27, 1985.

[MN07] Mitsuru Matsui and Junko Nakajima. On the power of bitslice implementation on intel core 2 processor. In Pascal Paillier and Ingrid Verbauwhede, editors, CHES 2007, volume 4727 of LNCS, pages 121-134. Springer, Heidelberg, September 2007.

[MPP09] Marine Minier, Raphael C.-W. Phan, and Benjamin Pousse. Distinguishers for ciphers and known key attack against Rijndael with large blocks. In Bart Preneel, editor, AFRICACRYPT 09, volume 5580 of $L N C S$, pages 60-76. Springer, Heidelberg, June 2009.

[MRST09] Florian Mendel, Christian Rechberger, Martin Schläffer, and Søren S. Thomsen. The rebound attack: Cryptanalysis of reduced Whirlpool and Grøstl. In Orr Dunkelman, editor, FSE 2009, volume 5665 of LNCS, pages 260-276. Springer, Heidelberg, February 2009.

[Nak08] Jorge Nakahara Jr. 3D: A three-dimensional block cipher. In Matthew K. Franklin, Lucas Chi Kwong Hui, and Duncan S. Wong, editors, CANS 08, volume 5339 of $L N C S$, pages 252-267. Springer, Heidelberg, December 2008.

[Nat01] National Institute of Standards and Technology. SP 800-38A: Recommendation for Block Cipher Modes of Operation: Methods and Techniques, December 2001.

[Nat16] National Institute of Standards and Technlology. Submission requirements and evaluation criteria for the post-quantum cryptography standardization process, 2016. https://csrc.nist.gov/ CSRC/media/Projects/Post-Quantum-Cryptography/documents / call-for-proposals-final-dec-2016.pdf.

[Nat18] National Academies of Sciences, Engineering, and Medicine. Quantum Computing: Progress and Prospects. The National Academies Press, Washington, DC, 2018.

[NC02] Michael A Nielsen and Isaac Chuang. Quantum computation and quantum information. AAPT, 2002.

[NP07] Jorge Nakahara Jr. and Ivan Carlos Pavão. Impossible-differential attacks on large-block Rijndael. In Juan A. Garay, Arjen K. Lenstra, Masahiro Mambo, and René Peralta, editors, ISC 200\%, volume 4779 of LNCS, pages 104-117. Springer, Heidelberg, October 2007.

[PGV94] Bart Preneel, René Govaerts, and Joos Vandewalle. Hash functions based on block ciphers: A synthetic approach. In Douglas R. Stinson, editor, CRYPTO'93, volume 773 of $L N C S$, pages 368-378. Springer, Heidelberg, August 1994.

[RBH17] Sondre Rønjom, Navid Ghaedi Bardeh, and Tor Helleseth. Yoyo tricks with AES. In Tsuyoshi Takagi and Thomas Peyrin, editors, ASIACRYPT 2017, Part I, volume 10624 of $L N C S$, pages 217-243. Springer, Heidelberg, December 2017. 
[Sas10] Yu Sasaki. Known-key attacks on Rijndael with large blocks and strengthening ShiftRow parameter. In Isao Echizen, Noboru Kunihiro, and Ryôichi Sasaki, editors, IWSEC 10, volume 6434 of $L N C S$, pages 301-315. Springer, Heidelberg, November 2010.

[Sho94] Peter W. Shor. Algorithms for quantum computation: Discrete logarithms and factoring. In 35th FOCS, pages 124-134. IEEE Computer Society Press, November 1994.

[Sim94] Daniel R. Simon. On the power of quantum computation. In 35th FOCS, pages 116-123. IEEE Computer Society Press, November 1994.

[SJS16] Vladimir Soukharev, David Jao, and Srinath Seshadri. Post-quantum security models for authenticated encryption. In Tsuyoshi Takagi, editor, Post-Quantum Cryptography - 7th International Workshop, PQCrypto 2016, pages 64-78. Springer, Heidelberg, 2016.

$\left[\mathrm{SLG}^{+} 16\right]$ Bing Sun, Meicheng Liu, Jian Guo, Longjiang Qu, and Vincent Rijmen. New insights on AES-like SPN ciphers. In Matthew Robshaw and Jonathan Katz, editors, CRYPTO 2016, Part I, volume 9814 of LNCS, pages 605-624. Springer, Heidelberg, August 2016.

[SY17] Fang Song and Aaram Yun. Quantum security of NMAC and related constructions - PRF domain extension against quantum attacks. In Jonathan Katz and Hovav Shacham, editors, CRYPTO 2017, Part II, volume 10402 of LNCS, pages 283-309. Springer, Heidelberg, August 2017.

[UDI $\left.{ }^{+} 11\right]$ Markus Ullrich, Christophe De Cannière, Sebastian Indesteege, Özgül Küçük, Nicky Mouha, and Bart Preneel. Finding optimal bitsliced implementations of 4x4-bit s-boxes. In SKEW 2011, 2011. Available at http://skew2011.mat. $\mathrm{dtu} . \mathrm{dk} / \mathrm{proceedings/.}$

[WGR ${ }^{+}$13] Qingju Wang, Dawu Gu, Vincent Rijmen, Ya Liu, Jiazhe Chen, and Andrey Bogdanov. Improved impossible differential attacks on large-block Rijndael. In Taekyoung Kwon, Mun-Kyu Lee, and Daesung Kwon, editors, ICISC 12, volume 7839 of LNCS, pages 126-140. Springer, Heidelberg, November 2013.

[Zha15] Mark Zhandry. A note on the quantum collision and set equality problems. Quantum Information \& Computation, 15(7\&8):557-567, 2015.

[Zha18] Mark Zhandry. How to record quantum queries, and applications to quantum indifferentiability. Cryptology ePrint Archive, Report 2018/276, 2018. https: //eprint.iacr.org/2018/276.

$\left[\mathrm{ZWP}^{+}\right.$08] Lei Zhang, Wenling Wu, Je Hong Park, Bon Wook Koo, and Yongjin Yeom. Improved impossible differential attacks on large-block Rijndael. In Tzong-Chen $\mathrm{Wu}$, Chin-Laung Lei, Vincent Rijmen, and Der-Tsai Lee, editors, ISC 2008, volume 5222 of $L N C S$, pages 298-315. Springer, Heidelberg, September 2008. 


\section{A Some Quantum Computing Notions}

Quantum Computing and Oracles We refer to [NC02] for a comprehensive introduction to quantum computing. While it is certainly difficult to assert the effective time of yet-tobe-implemented quantum operations, we use as common ground the quantum circuit model. Quantum and classical time complexities can only be compared roughly; but two quantum circuits, as they are written in the same abstract manner, can be compared precisely. By replacing classical exhaustive search by quantum exhaustive search, i.e. Grover's search, the notions of quantum level of security, quantum security margin and quantum attack become immediately available, by analogy with their classical counterparts.

A quantum circuit consists of a sequence of operations (or quantum gates) applied to a pool of qubits. Qubits are the analogue of classical bits. Classically, the state of a bit is either 0 or 1. Quantumly, the state of a qubit is a vector in a two-dimensional Hilbert space, with a canonical basis $\{|0\rangle,|1\rangle\}$. The qubits are first prepared in an arbitrary state, say $|0\rangle$. The operations, quantum gates and oracle queries, are all unitary operators; and they are all reversible. Via entanglement, a general $n$-qubit system can only be described on a basis of $2^{n}$ vectors. But all information on the system is not accessible. The different basis vectors $|i\rangle$ have complex amplitudes $\alpha_{i}$, such that $\left|\alpha_{i}\right|^{2}$ is the probability to obtain $i$ upon measurement of the system. A measurement destroys the state (the superposition collapses) and replaces it with $|i\rangle$ for the obtained $i$. Consequently, only $n$ bits of information can be extracted from an $n$-qubit quantum system. The sequence of quantum gates causes constructive and destructive interferences between the states, which reduce the amplitude of "bad states" and increase that of "good states", so that, upon measurement, we expect a meaningful result.

Throughout this document, we use the circuit model. We do not need to specify exactly the universal gate set used, as regarding quantum attacks, our complexities will be given in multiples of a quantum circuit for SATURNIN.

Oracles. Classically, an oracle (for encryption, decryption, verification...) can be seen as a black-box function $f$ which takes an adversary-controlled input $x$ and returns $f(x)$. Quantumly, a superposition oracle is a unitary $O_{f}$ which takes an input $|x\rangle|b\rangle$ and returns $|x\rangle|b \oplus f(x)\rangle$. The output $f(x)$ is only written on the additional register, ensuring reversibility. The input state can be in any superposition, but this does not make the adversary in power to compute "all possibilities at once", for example to query a secret-key oracle on all the codebook in one query, since the information can only obtained by measurement, and a measurement makes the superposition collapse.

Simon's algorithm. Given superposition query access to a function $f:\{0,1\}^{n} \rightarrow\{0,1\}^{n}$ which "hides" a period $s$, i.e. $f(x \oplus s)=f(x)$ for all $s$, Simon's algorithm [Sim94] recovers $s$ using $O(n)$ queries to $O_{f}$ and little (polynomial in $n$ ) computation overhead.

Grover's algorithm. Given superposition query access to a function $f:\{0,1\}^{n} \rightarrow\{0,1\}$, such that $f^{-1}(1)$ contains $2^{t}$ elements, Grover's algorithm [Gro96] recovers a preimage of 1 in $O\left(2^{(n-t) / 2}\right)$ time and queries to $O_{f}$ instead of $O\left(2^{n-t}\right)$ classical time and queries to $f$. This algorithm consists in iterating $O\left(2^{(n-t) / 2}\right)$ times a unitary operator which uses a query to $O_{f}$ to move some amplitude towards the elements of $f^{-1}(1)$.

BHT Collision Search Algorithm. The algorithm of [BHT98] uses Grover's search as a subroutine. It makes $2^{n / 3}$ queries, stores them in quantum hardware, and looks for a collision on one of the queried elements. The probability for a random element to collide on this table is $\frac{2^{n / 3}}{2^{n}}$, so this Grover's search step requires time $2^{n / 3}$. This algorithm is optimal for a random function. 


\section{B Impossible Differential Distinguishers}

We reproduce here the two impossible differential distinguishers that we have studied for SAturnin. The whole cubic state of the cipher is represented as its 4 slices. We denote by $\mathrm{S}$ the $\mathrm{S}-$ Box layer, $\mathrm{MD}$ the operation $\mathrm{SR}_{\text {slice }} \circ \mathrm{MC} \circ \mathrm{SR}_{\text {slice }}^{-1}$ and by $\mathrm{MS}$ the operation $\mathrm{SR}_{\text {sheet }} \circ \mathrm{MC} \circ \mathrm{SR}_{\text {sheet }}^{-1}$. 

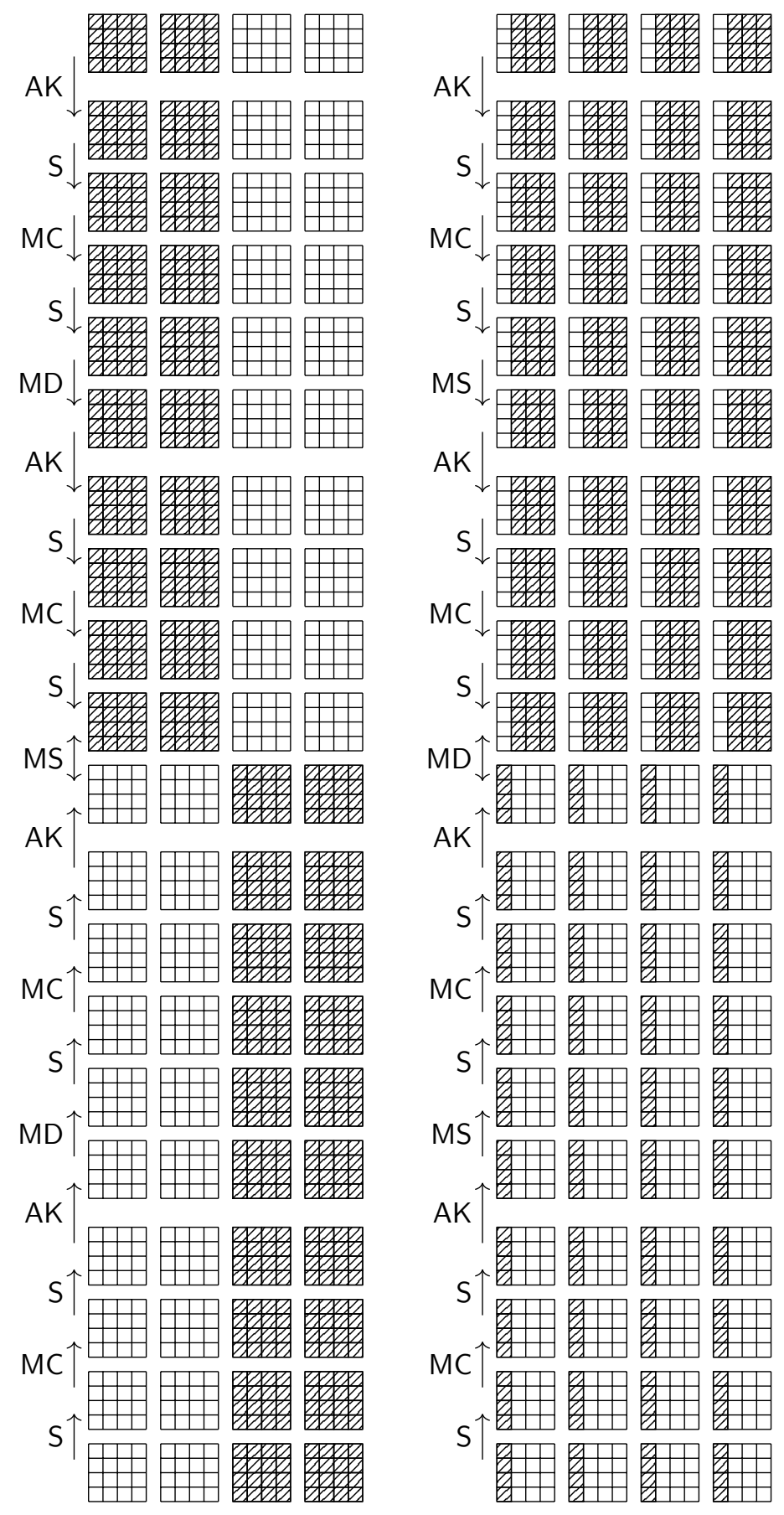

Figure 21: Paths A (left) and B (right). 


\section{On the Name}

The transcription of the French pronounciation of "SATURNIN" is given in Figure 22.

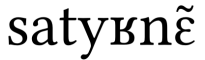

Figure 22: How to pronounce SATURNIN using the International Phonetic Alphabet.

There are multiple motivations for this name.

Saturnin the Duck. The duck is undeniably a symbol of lightness because it floats. It has been famously used as the reference for lightness throughout the ages, for instance by Sir Bedevere [GJ75]. The bantamweight weight class in boxing is also named after a small duck, and corresponds to lightweight fighters. As it turns out, Saturnin is the most famous duck in France: it was the hero of a well-known TV show ${ }^{6}$. A Saturnin-like ${ }^{7}$ yellow duck is shown in Figure 23a.

Kepler's Mysterium Cosmographicum. The astronomer made the following observation in his 1596 opus, as described on his wikipedia page:

[Kepler] found that each of the five Platonic solids could be inscribed and circumscribed by spherical orbs; nesting these solids, each encased in a sphere, within one another would produce six layers, corresponding to the six known planets-Mercury, Venus, Earth, Mars, Jupiter, and Saturn. By ordering the solids selectively - octahedron, icosahedron, dodecahedron, tetrahedron, cube-Kepler found that the spheres could be placed at intervals corresponding to the relative sizes of each planet's path, assuming the planets circle the Sun.

This system is summarized in Figure 23b. As we can see, the planet Saturn is associated with the cube - the exact shape of our cipher.

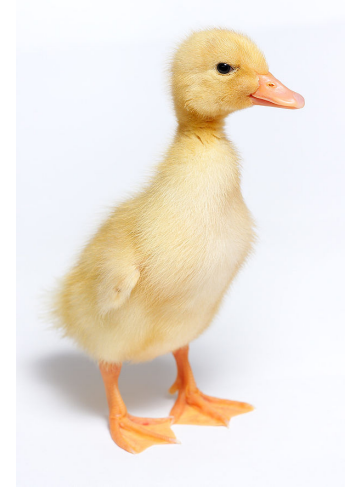

(a) A Saturnin-like duck (credit: Fir0002/Flagstaffotos).

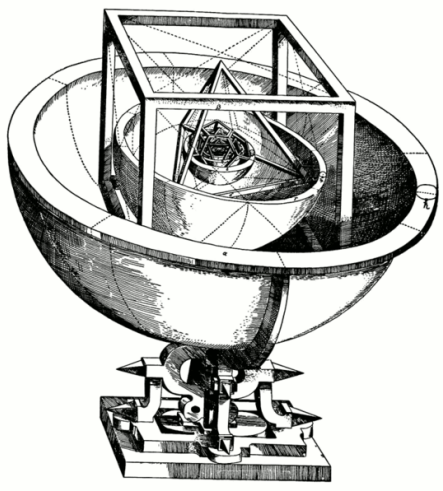

(b) From Kepler's Mysterium Cosmographicum, via Wikipedia.

Figure 23: Satunin pictures

Wisdom. The planet Saturn is a symbol of the wisdom coming with age, a fitting metaphor for our reliance on the knowledge accumulated since the publication of the AES.

\footnotetext{
${ }^{6}$ https://fr.wikipedia.org/wiki/Les_Aventures_de_Saturnin.

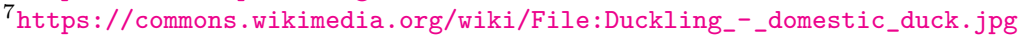

